\title{
MAPPING CLASS GROUPS AND MODULI SPACES OF CURVES
}

\author{
RICHARD HAIN AND EDUARD LOOIJENGA
}

\section{Contents}

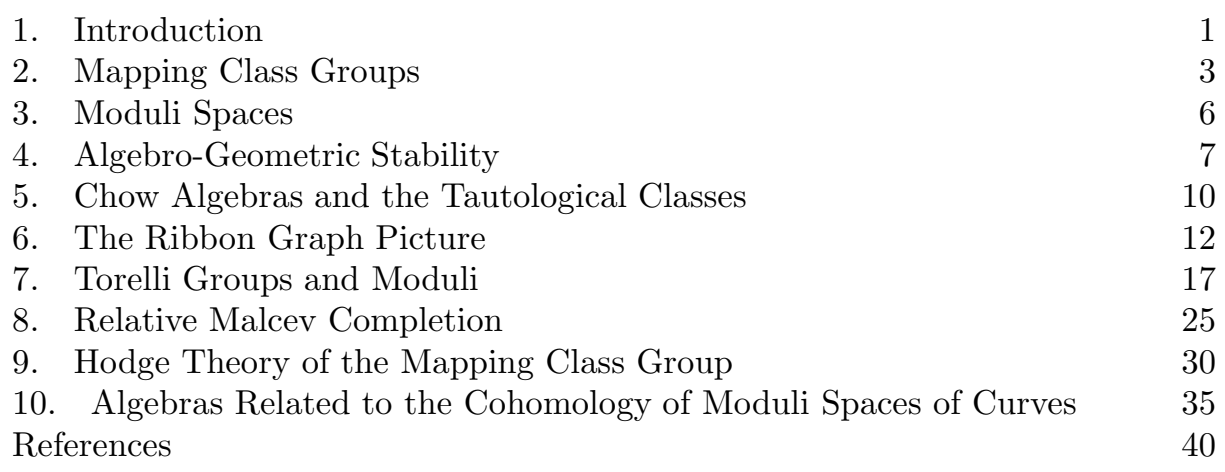

\section{INTRODUCTION}

It is classical that there is a very strong relation between the topology of $\mathcal{M}_{g}$, the moduli space of smooth projective curves of genus $g$, and the structure of the mapping class group $\Gamma_{g}$, the group of homotopy classes of orientation preserving diffeomorphisms of a compact orientable surface of genus $g$. The geometry of $\mathcal{M}_{g}$, the topology of $\mathcal{M}_{g}$, and the structure of $\Gamma_{g}$ are all intimately related. Until recently, the principal tools for studying these topics were Teichmüller theory (complex analysis and hyperbolic geometry), algebraic geometry, and geometric topology. Recently, a fourth cornerstone has been added, and that is physics which enters through the theories of quantum gravity and conformal field theory. Already these new ideas have had a remarkable impact on the subject through the ideas of Witten and the work of Kontsevich. In this article, we survey some recent developments in the understanding of moduli spaces. Some of these are classical (do not use physical ideas), while others are modern. One message we would like to convey is that algebraic geometers, topologists, and physicists who work on moduli spaces of curves may have a lot to learn from each other.

Having said this, we should immediately point out that, partly due to our own limitations, there are important developments that we have not included in this survey. Our most notable omission is the arithmetic the aspect of the theory, much of which originates in Grothendieck's fundamental works [19], [20]. We direct readers to the volume [21] and to the recent papers of Ihara, Nakamura and Oda for other

Supported in part by grants from the National Science Foundation. 
recent developments. Other topics we have not covered include conformal field theory and recent work of Ivanov [38] and Ivanov and McCarthy [39] on homomorphisms from mapping class groups and arithmetic groups to mapping class groups. Of particular importance is Ivanov's version of Margulis rigidity for mapping class groups [38] which he obtains using some recent fundamental work of Kaimanovich and Masur [17] on the ergodic theory of Teichmüller space.

We shall denote the moduli space of $n$ pointed smooth projective curves of genus $g$ by $\mathcal{M}_{g}^{n}$. Knudsen, Mumford and Deligne constructed a canonical compactification $\overline{\mathcal{M}}_{g}^{n}$ of it. It is the moduli space of stable $n$ pointed projective curves of genus $g$. It is projective variety with only finite quotient singularities. Perhaps the most important developments of the decade concern the Chow rings] of $\mathcal{M}_{g}^{n}$ and $\overline{\mathcal{M}}_{g}^{n}$.

The first Chern class of the relative tangent bundle of the universal curve associated to the $i$ th point is a class $\bar{\tau}_{i}$ in $\mathrm{CH}^{1}\left(\overline{\mathcal{M}}_{g}^{n}\right)$. One can consider monomials in the $\bar{\tau}_{i}$ 's of polynomial degree equal to the dimension of some $\overline{\mathcal{M}}_{g}^{n}$. For such a monomial, one can take the degree of the monomial as a zero cycle on $\overline{\mathcal{M}}_{g}^{n}$ to obtain a rational number. These can be assembled into a generating function. Witten conjectured that this formal power series satisfies a system of partial differential operators. Kontsevich proved this using topological arguments, and thereby provided inductive formulas for these intersection numbers. These developments are surveyed in Section 6 .

For each postive integer $i$, Mumford defined a tautological class $\bar{\kappa}_{i}$ in $\mathrm{CH}^{i}\left(\overline{\mathcal{M}}_{g}\right)$. The restrictions $\kappa_{i}$ of these classes to $\mathrm{CH}^{\bullet}\left(\mathcal{M}_{g}\right)$ generate a subalgebra of $\mathrm{CH}^{\bullet}\left(\mathcal{M}_{g}\right)$ which is called the tautological algebra of $\mathcal{M}_{g}$. Faber has conjectured that this ring has the structure of the $(p, p)$ part of the cohomology ring of a smooth complex projective variety of complex dimension $g-2$. That is, it satisfies Poincaré duality and has the "Hard Lefschetz Property" with respect to $\kappa_{1}$. Considerable evidence now exists for this conjecture, much of which is presented in Section 5 . Other developments on the Chow ring, such as explicit computations in low genus, are also surveyed there.

In the early 80s, Harer proved that the cohomology in a given degree of the $\mathcal{M}_{g}$ is independent of the genus once the genus is sufficiently large relative to the degree. These stable cohomology groups form a graded commutative algebra which is known to be free. The tautological classes $\kappa_{i}$ freely generate a polynomial algebra inside the stable cohomology ring. Mumford and others have conjectured that the stable cohomology of the $\mathcal{M}_{g}$ is generated by the $\kappa_{i}$ 's. Some progress has been made towards this conjecture which we survey throughout the paper. In Section 4 we consider the stabilization maps from an algebro-geometric point of view, and in Section 10 we survey Kontsevich's methods for constructing classes in the cohomology of the $\mathcal{M}_{g}^{n}$.

We have also tried to advertise the fecund work of Dennis Johnson on the Torelli groups. The Torelli group $T_{g}$ is the subgroup of the mapping class group $\Gamma_{g}$ consisting of those diffeomorphism classes that act trivially on the homology of the reference surface. This mysterious group, in some sense, measures the difference between curves and abelian varieties and appears to play a subtle role in the geometry of $\mathcal{M}_{g}$. Johnson proved that $T_{g}$ is finitely generated when $g \geq 3$ and computed

\footnotetext{
${ }^{1}$ All Chow rings and cohomology groups in this paper are with $\mathbb{Q}$ coefficients except when explicit coefficients are used.
} 
its first integral homology group. These computations have direct geometric applications, especially when combined with M. Saito's work in Hodge theory - for example, they restrict the normal functions defined over $\mathcal{M}_{g}$ and its standard level covers. From this, one can give a computation of the Picard group of the generic curve with a level $l$ structure. Johnson's work and its applications is surveyed in Section 7.

Since $\Gamma_{g}$ is the orbifold fundamental group of $\mathcal{M}_{g}$, an algebraic variety, one should be able to apply Hodge theory and Galois theory to study its structure. In Section 9 we survey recent work on applications of Hodge theory to understanding the structure of the Torelli groups, mainly via its Malcev completion. In Section 10 we combine this Hodge theory with a recent result of Kawazumi and Morita to show that some of the classes in the cohomology of $\mathcal{M}_{g}$ constructed by Kontsevich using graph cohomology are, after stabilization, polynomials in the $\kappa_{i}$ 's. Thus Hodge theory provides some evidence for the belief (often referred to as Mumford's conjecture) that the stable cohomology of the mapping class group is generated by the $\kappa_{i}$ 's.

Notation and Conventions. All varieties will be defined over $\mathbb{C}$ unless explicitly stated to the contrary. Unless explicit coefficients are used, all (co)homology groups are with rational coefficients. We will often abreviate mixed Hodge structure by MHS. The sub- or superscript $p r$ on a (co)homology group will denote the primitive part in both the context of the Hard Lefschetz Theorem and in the context of Hopf algebras.

Acknowledgements. We would like to thank Carel Faber for his comments on part of an earlier version of this paper and Shigeyuki Morita for explaining to us some of his recent work. We gratefully acknowledge support by the AMS that enabled us to attend this conference.

\section{Mapping Class Groups}

Fix a compact connected oriented reference surface $S_{g}$ of genus $g$, and a sequence of distinct points $\left(x_{0}, x_{1}, x_{2}, \ldots\right)$ in $S_{g}$. Let us write $S_{g}^{n}$ for the open surface $S$ $\left\{x_{1}, \ldots, x_{n}\right\}$ and $\pi_{g}^{n}$ for its fundamental group $\pi_{1}\left(S_{g}^{n}, x_{0}\right)$. This group admits a presentation with generators $\alpha_{ \pm 1}, \ldots, \alpha_{ \pm g}, \beta_{1}, \ldots, \beta_{n}$ and relation

$$
\left(\alpha_{1}, \alpha_{-1}\right) \cdots\left(\alpha_{g}, \alpha_{-g}\right)=\beta_{1} \cdots \beta_{n} .
$$

(For $n=0$ the righthand side is to be interpreted as the unit element.) The generators are represented by loops that do not meet outside the base point; $\beta_{i}$ is represented by a loop that follows an arc to a point close to $x_{i}$, makes a simple loop around $x_{i}$, and returns to the base point along the same arc.

Let $\operatorname{Diff}^{+}(S)_{r}^{n}$ denote the group of orientation preserving diffeomorphisms of $S$ that fix the $x_{i}$ for $i=1, \ldots, n+r$, and are the identity on $T_{x_{i}} S$ for $i=n+$ $1, \ldots, n+r$. Although not really necessary at this stage, it is convenient to assume that $2 g-2+n+2 r>0$. In other words, we do not consider the cases where $(g, n, r)$ is $(0,1,0),(0,0,1),(0,2,0)$ or $(1,0,0)$. We will keep this assumption throughout the paper.

The mapping class group $\Gamma_{g, r}^{n}$ is defined to be the group of connected components of this group:

$$
\Gamma_{g, r}^{n}=\pi_{0} \operatorname{Diff}^{+}(S)_{r}^{n}
$$


We omit the decorations $n$ and $r$ when they are zero. The mapping class group $\Gamma_{g}^{n}$ acts on $\pi_{g}^{n}$ by outer automorphisms. A theorem that goes back to Baer (1928) and Nielsen (1927) 72 identifies $\Gamma_{g}$, via this representation, with the subgroup of $\operatorname{Out}\left(\pi_{g}\right)$ (of index two) that acts trivially on $H_{2}\left(\pi_{g}\right) \cong H_{2}\left(S_{g}\right)$.

If $n \geq 1$, then consider the diagonal action of $\operatorname{Aut}\left(\pi_{g}^{n}\right)$ on $\left(\pi_{g}^{n}\right)^{n}$. Clearly, $\operatorname{Out}\left(\pi_{g}^{n}\right)$ acts on the set of orbits of $\pi_{g}^{n}$ (acting by inner automorphisms on each component) in $\left(\pi_{g}^{n}\right)^{n}$. Now $\Gamma_{g}^{n}$ can be identified with the group of outer automorphisms of $\pi_{g}^{n}$ that preserve the image of $\left(\beta_{1}, \ldots, \beta_{n}\right)$ in $\pi_{g}^{n} \backslash\left(\pi_{g}^{n}\right)^{n}$. If we choose $x_{n}$ as a base point, then a corresponding assertion holds: $\Gamma_{g}^{n}$ can be identified with a subgroup of $\operatorname{Aut}\left(\pi_{1}\left(S_{g}^{n-1}, x_{n}\right)\right)$ that is characterized in a similar way. The evident homomorphism $\Gamma_{g}^{n} \rightarrow \Gamma_{g}^{n-1}$ is surjective and its kernel can be identified with $\pi_{1}\left(S_{g}^{n-1}, x_{n}\right)$ (acting by inner automorphisms). Ivanov and McCarthy [39] recently showed that the resulting exact sequence cannot be split.

2.1. Generators and basic properties. Although a lot is known about these groups they are still poorly understood. Let us quickly review some of their basic properties. Dehn proved in [10] that the mapping class groups are generated by the 'twists' that are now named after him: if $\alpha$ is a simple (unoriented) loop on $S_{g}^{n+r}$, then parameterize a regular neighborhood of $\alpha$ in $S_{g}^{n+r}$ by the cylinder $[0,1] \times S^{1}$ (preserving orientations) and define an automorphism of $S_{g}$ that on this neighborhood is given by $(t, z) \mapsto\left(t, e^{2 \pi i t} z\right)$ and is the identity elsewhere. The isotopy class of this automorphism only depends on the isotopy class of $\alpha$ and is called the Dehn twist along $\alpha$. (Perhaps we should add that $\alpha$ is, in turn, already determined by its free homotopy class, in other words, by the associated conjugacy class in $\pi_{g}^{n}$.) The corresponding element of $\Gamma_{g, r}^{n}$ is the identity precisely when $\alpha$ bounds a disk in $S_{g}-\left\{x_{n+1}, \ldots, x_{n+r}\right\}$ which meets $\left\{x_{1}, \ldots, x_{n}\right\}$ in at most one point. Several people have found a finite presentation for the mapping class groups. One with few generators was given by Waynryb [78]. From this representation one recovers the well known fact that the mapping class groups considered here are perfect when $g \geq 3$.

There is an obvious homomorphism $\Gamma_{g, n} \rightarrow \Gamma_{g}^{n}$. It is easy to see that it is surjective and that the kernel is generated by the Dehn twists around the points $x_{1}, \ldots, x_{n}$. These Dehn twists generate a free abelian central subgroup of $\Gamma_{g}^{n}$ of rank $n$. Now recall that a central extension of a discrete group $G$ by $\mathbb{Z}$ determines an extension class in $H^{2}(G, \mathbb{Z})$; it has a geometric interpretation as a first Chern class. In the present case we have $n$ such classes $\tau_{i} \in H^{2}\left(\Gamma_{g}^{n} ; \mathbb{Z}\right), i=1, \ldots, n$.

Conversely, each subgroup of $H^{2}(G, \mathbb{Z})$ determines a central extension of $G$ by that subgroup. Harer proved that $H^{2}\left(\Gamma_{g, r}\right)$ is infinite cyclic if $g \geq 3$ [27], so that there is a corresponding central extension

$$
0 \rightarrow \mathbb{Z} \rightarrow \tilde{\Gamma}_{g, r} \rightarrow \Gamma_{g, r} \rightarrow 1
$$

Since $H_{1}\left(\Gamma_{g, r}\right)$ vanishes, this central extension is perfect (and universal). A nice presentation of it was recently given by Gervais 16. The (imperfect) central extension by $\frac{1}{12} \mathbb{Z}$ containing this extension appears in the theory of conformal blocks; it has a simple geometric description which we will give in Section 3 .

2.2. Stable cohomology. The mapping class groups $\Gamma_{g, r}^{n}$ turn up in a connected sum construction that we describe next. It is convenient to do this in a somewhat abstract setting. Suppose we are given a closed, oriented (but not necessarily 
connected) surface $S$, a finite subset $Y \subset S$, and a fixed point free involution $\iota$ of $Y$. Assume that $\iota$ has been lifted to an orientation reversing linear involution $\tilde{\iota}$ on the spaces of rays $\operatorname{Ray}(T S \mid Y)$. The real oriented blow up $S_{Y} \rightarrow S$ is a surface with boundary canonically isomorphic to $\operatorname{Ray}(T S \mid Y)$. So $\tilde{\iota}$ defines an orientation reversing involution of this boundary. Welding the boundary components of $S_{Y}$ by means of this involution produces a closed surface $S(\tilde{\iota})$. Some care is needed to give it a differentiable structure inducing the given one on $S_{Y}$. Although there is no unique way to do this, all natural choices lie in the same isotopy class. If $S$ happens to have a conformal structure, then each choice of a real ray $L$ in $T_{p} S \otimes_{\mathbb{C}} T_{\iota(p)} S$ determines a lift of $\iota$ over the pair $\{p, \iota(p)\}$ : if $l$ is a ray in $T_{p} S$, then $\tilde{\iota}(l)$ is determined uniquely by the condition $l \otimes_{\mathbb{C}} \tilde{\iota}(l)=L$.

If $S(\tilde{\iota})$ is connected, then each finite subset $X \subset S-Y$ determines a natural homomorphism from the mapping class group which is perhaps best denoted by $\Gamma(S)_{Y}^{X}$ (a product of groups of the type $\Gamma_{g, r}^{n}$ ) to the mapping class group $\Gamma(S(\tilde{\iota}))^{X}$. The image of this homomorphism is simply the stabilizer of the simple loops indexed by $Y / \iota$ that are images of boundary components of $S_{Y}$. Its kernel is a free abelian group whose generators can be labeled by a system of representatives $R$ of $\iota$ orbits in $Y$. Indeed, take for any element $y \in R$, the composite of the Dehn twist around $y$ and the inverse of the Dehn twist around $\iota(y)$. These maps appear in the stability theorems and are at the root of the recent operad theoretic approaches to the study of the cohomology of mapping class groups.

Theorem 2.1 (Stability theorem, Harer [29]). There exists a positive constant $c$ with the following property. If $S(\tilde{\iota})$ is connected and $S^{\prime}$ is a connected component of $S$ and $X$ a finite subset of $S^{\prime} \backslash Y$, then the homomorphism

$$
\Gamma\left(S^{\prime}\right)_{Y \cap S^{\prime}}^{X} \rightarrow \Gamma(S(\tilde{\iota}))^{X}
$$

induces an isomorphism on integral cohomology in degree $\leq$ c.genus $\left(S^{\prime}\right)$.

The constant $c$ appearing in this theorem was $1 / 3$ in Harer's original paper. It was later improved to $1 / 2$ by Ivanov in [35]. Most recently, Harer [31] has showed that we can take $c$ to be about $2 / 3$ and that this is the minimal possible value. There is also a version for twisted coefficients, due to Ivanov [36].

Harer's theorem says essentially that the $k$ th cohomology group of $\Gamma_{g, r}^{n}$ depends only on $n$, provided that $g$ is large enough. These stable cohomology groups are the cohomology of a single group, namely the group $\Gamma_{\infty}^{n}$ of compactly supported mapping classes of a surface $S_{\infty}$ of infinite genus (with one end, say) that fix a given set of $n$ distinct points.

Among the homomorphisms defined above are maps $\Gamma_{g, 1} \times \Gamma_{g^{\prime}, 1} \rightarrow \Gamma_{g+g^{\prime}}$. These stabilize and define homomorphisms of $\mathbb{Q}$ algebras

$$
\mu: H^{\bullet}\left(\Gamma_{\infty}\right) \rightarrow H^{\bullet}\left(\Gamma_{\infty}\right) \otimes H^{\bullet}\left(\Gamma_{\infty}\right) .
$$

This defines a coproduct on $H^{\bullet}\left(\Gamma_{\infty}\right)$. Together with the cup product, this gives $H^{\bullet}\left(\Gamma_{\infty}\right)$ the structure of a connected graded-bicommutative Hopf algebra. The classification of such Hopf algebras implies that $H^{\bullet}\left(\Gamma_{\infty}\right)$ is free as a graded algebra and is generated by its set of primitive elements

$$
H_{\mathrm{pr}}^{\bullet}\left(\Gamma_{\infty}\right):=\left\{x \in H^{+}\left(\Gamma_{\infty}\right): \mu(x)=x \otimes 1+1 \otimes x\right\} .
$$

For each $i>0$, Mumford [69] and Morita [63] independently found a class $\kappa_{i}$ in $H_{\mathrm{pr}}^{2 i}\left(\Gamma_{\infty}\right)$ (we shall recall the definition in Section 4 ) and Miller 61 showed that each $\kappa_{i}$ is nonzero, and therefore that the $\kappa_{i}$ generate a polynomial subalgebra of the 
stable cohomology. Mumford conjectured that these classes span all of $H_{\mathrm{pr}}^{\bullet}\left(\Gamma_{\infty}\right)$. This has been verified by Harer in a series of papers [27], 30], [32] in degrees $\leq 4$.

The first Chern class $\tau_{i} \in H^{2}\left(\Gamma_{g}^{n} ; \mathbb{Z}\right)$ stabilizes also and we may think of it as an element of $H^{\bullet}\left(\Gamma_{\infty}^{n} ; \mathbb{Z}\right)(i=1, \ldots, n)$. The forgetful map $\Gamma_{\infty}^{n} \rightarrow \Gamma_{\infty}$ gives $H^{\bullet}\left(\Gamma_{\infty}^{n}\right)$ the structure of a module over this Hopf algebra.

From the stability theorem one can deduce:

Theorem 2.2 (Looijenga [58]). The algebra $H^{\bullet}\left(\Gamma_{\infty}^{n} ; \mathbb{Z}\right)$ is freely generated by the classes $\tau_{1}, \ldots, \tau_{n}$ as a graded-commutative $H^{\bullet}\left(\Gamma_{\infty} ; \mathbb{Z}\right)$ algebra.

\section{Moduli Spaces}

A conformal structure and an orientation on $S_{g}$ determine a complex structure on $S_{g}$. The Teichmüller space $\mathcal{X}_{g, r}^{n}$ is the space of conformal structures on $S_{g}$ (with some reasonable topology) up to isotopies that fix $\left\{x_{1}, \ldots, x_{n+r}\right\}$ pointwise and act trivially on the tangent spaces $T_{x_{i}} S$ for $i=n+1, \ldots, n+r$. It is in a natural way a complex manifold of dimension $3 g-3+n+2 r$. As a real manifold it is diffeomorphic to a cell. The group $\Gamma_{g, r}^{n}$ acts naturally on it. This action is properly discontinuous and a subgroup of finite index acts freely.

If $\Gamma$ is any subgroup of $\Gamma_{g, r}^{n}$ that acts freely, then the orbit space $\Gamma \backslash \mathcal{X}_{g, r}^{n}$ is a classifying space for $\Gamma$ and so its singular integral cohomology coincides with $H^{\bullet}(\Gamma ; \mathbb{Z})$. This is even true with twisted coefficients: if $V$ is a $\Gamma$ module, then the trivial sheaf over $\mathcal{X}_{g, r}^{n}$ with fiber $V$ comes with an obvious (diagonal) action of $\Gamma$. Passing to $\Gamma$ orbits yields a locally constant sheaf $\mathbb{V}$ on $\Gamma \backslash \mathcal{X}_{g, r}^{n}$. The cohomology of this sheaf equals $H^{\bullet}(\Gamma ; V)$. For an arbitrary subgroup $\Gamma$ of $\Gamma_{g, r}^{n}$ these statements still hold as long as we take our coefficients to be $\mathbb{Q}$ vector spaces (but $\mathbb{V}$ need no longer be locally constant). For $\Gamma=\Gamma_{g, r}^{n}$, we denote the orbit space by $\mathcal{M}_{g, r}^{n}$.

The space $\mathcal{M}_{g, r}^{n}$ is, in a natural way, a normal analytic space and the obvious forgetful maps such as $\mathcal{M}_{g, r}^{n} \rightarrow \mathcal{M}_{g}^{n}$ are analytic. An interpretation as a coarse moduli space makes it possible to lift this analytic structure to the algebraic category. To see this, we first choose a nonzero vector in each tangent space $T_{x_{i}} S_{g}$. Each triple $(C ; x, v)$, where $C$ is a connected nonsingular complex projective curve $C$ of genus $g, x$ an injective map $x:\{1, \ldots, n+r\} \rightarrow C$, and $v$ a nowhere zero section of $T C$ over $\{n+1, \ldots, n+r\}$, determines an element of $\mathcal{M}_{g, r}^{n}$. This point depends only on the isomorphism class of $(C, x, v)$ with respect to the obvious notion of isomorphism. Since each conformal structure on $S$ gives $S$ the structure of a nonsingular complex projective curve, $\mathcal{M}_{g, r}^{n}$ can be identified can be identified with the space of isomorphism classes of such triples. From the work of Knudsen, Mumford and Deligne, we know that $\mathcal{M}_{g}^{n}$ is, in a natural way, a quasi-projective orbifold. Recall that they also constructed a projective completion $\overline{\mathcal{M}}_{g}^{n}$ of $\mathcal{M}_{g}^{n}$, the Deligne-Mumford completion [12], that also admits the interpretation of a coarse moduli space. Its points parameterize the connected stable $n$ pointed curves $(C, x)$ of arithmetic genus $g$, where we now allow $C$ to have ordinary double points, but still require $x$ to map to the smooth part of $C$ and the automorphism group of $(C, x)$ to be finite. The Deligne-Mumford boundary $\overline{\mathcal{M}}_{g}^{n}-\mathcal{M}_{g}^{n}$ is a normal crossing divisor in the orbifold sense. There is a projective morphism $\overline{\mathcal{M}}_{g}^{n+1} \rightarrow \overline{\mathcal{M}}_{g}^{n}$, defined by forgetting the last point. It comes with $n$ sections $x_{1}, \ldots, x_{n}$. The fibers of this morphism are stable $n$ pointed curves (modulo finite automorphism groups) and

\footnotetext{
${ }^{2} \mathrm{He}$ also tells us that he has checked that there are no stable primitive classes in degree 5.
} 
the morphism can be regarded as the universal stable $n$ pointed curve (in an orbifold sense). Let $\omega$ denote the relative dualizing sheaf of this morphism, considered as a line bundle in the orbifold sense. We can then think of $\mathcal{M}_{g, r}^{n}$ as the set of $\left(v_{n+1}, \ldots, v_{n+r}\right)$ in the total space of $x_{n+1}^{*} \omega \oplus \cdots \oplus x_{n+r}^{*} \omega \mid \mathcal{M}_{g}^{n+r}$ that have each component nonzero. So $\mathcal{M}_{g, r}^{n}$ is also quasi-projective.

Each finite quotient group $G$ of $\Gamma_{g}^{n}$ determines, in an obvious way, a Galois cover $\mathcal{M}_{g}^{n}[G] \rightarrow \mathcal{M}_{g}^{n}$. The Deligne-Mumford completion $\overline{\mathcal{M}}_{g}^{n}[G]$ of this cover is by definition the normalization of $\overline{\mathcal{M}}_{g}^{n}$ in $\mathcal{M}_{g}^{n}[G]$.

Theorem 3.1 (Looijenga [55]). There exists a finite group $G$ such that $\overline{\mathcal{M}}_{g}[G]$ is smooth with a normal crossing divisor as Deligne-Mumford boundary.

This has been extended by De Jong and Pikaart 74 to arbitrary characteristic, and by Boggi and Pikaart (independently) to the case $n>0$. (They show that it also can be arranged that each irreducible component of the Deligne-Mumford boundary of $\overline{\mathcal{M}}_{g}[G]$ is smooth.) This makes it relatively easy to define the Chow ring for $\overline{\mathcal{M}}_{g}^{n}$ : if $\overline{\mathcal{M}}_{g}^{n}[G]$ is smooth, then define $\mathrm{CH}^{\bullet}\left(\overline{\mathcal{M}}_{g}^{n}\right)$ to be the $G$ invariant part of $\mathrm{CH}^{\bullet}\left(\overline{\mathcal{M}}_{g}^{n}[G]\right.$ ) (we take algebraic cycles modulo rational equivalence with coefficients in $\mathbb{Q})$. It is easy to see that this is independent of the choice if $G$.

The central extension of $\Gamma_{g}(g \geq 3)$ discussed in Subsection 2.1 takes the geometric form of a complex line bundle over Teichmüller space with $\Gamma_{g}$ action and hence yields an orbifold line bundle over $\mathcal{M}_{g}$. Its twelfth tensor power has a concrete description: it is the determinant bundle of the direct image of the relatively dualizing sheaf of $\mathcal{M}_{g}^{1} \rightarrow \mathcal{M}_{g}$ (this is a rank $g$ vector bundle). The orbifold fundamental group of the associated $\mathbb{C}^{\times}$bundle is just the central extension of $\Gamma_{g}$ by $\frac{1}{12} \mathbb{Z}$ mentioned in Subsection 2.1.

Since $\Gamma_{g}$ is perfect when $g \geq 3$, we have $H^{1}\left(\Gamma_{g}\right)=0$. Ivanov has asked the following question:

Question 3.2 (Ivanov). Is it true that $H^{1}(\Gamma)$ vanishes for all finite index subgroups $\Gamma$ of $\Gamma_{g}$, at least when $g$ is sufficiently large?

This would imply that the Picard group of each finite unramified cover of $\mathcal{M}_{g}$ (in the orbifold sense) is finitely generated. The answer to Ivanov's questions is affirmative, for example, for subgroups of finite index of $\Gamma_{g}, g \geq 3$, that contain the Torelli group — see (7.4).)

\section{Algebro-Geometric Stability}

The Deligne-Mumford completion $\overline{\mathcal{M}}_{g}^{n}$ comes with a natural stratification into orbifolds, with each stratum parameterizing stable $n$ pointed curves of a fixed topological type $T$. Denote this stratum by $\mathcal{M}(T)$. It has codimension equal to the number of singular points of $T$. The normalization of the topological type $T$ is an oriented closed surface $S$ that comes with $n$ distinct numbered points $X=\left\{x_{1}, \ldots, x_{n}\right\}$ and a finite subset $Y$ of $S X$ with a fixed point free involution $\iota$, so that $T$ is recovered by identifying the points of $Y$ according to $\iota$. These topological data define a moduli space $\mathcal{M}(S)^{X \cup Y}$ of the same type (we hope that the notation is self-explanatory) and there is a natural morphism $\mathcal{M}(S)^{X \cup Y} \rightarrow \mathcal{M}(S / \iota)^{X}$ that is a Galois cover of orbifolds. This morphism extends to a finite surjective morphism from the Deligne-Mumford completion $\overline{\mathcal{M}}(S)^{X \cup Y}$ to the closure of $\mathcal{M}(T)$ in $\overline{\mathcal{M}}_{g}^{n}$. The resulting morphism $\overline{\mathcal{M}}(S)^{X \cup Y} \rightarrow \overline{\mathcal{M}}_{g}^{n}$ has only self-intersections of 
normal crossing type and so carries a normal bundle in the orbifold sense. This normal bundle is a direct product of line bundles with one summand for each $\iota$ orbit $\left\{p, p^{\prime}\right\}$, namely $p^{*} \omega^{-1} \otimes p^{*} \omega^{-1}$. (To see this, notice that the restriction of the universal curve to $\overline{\mathcal{M}}(T)$ has a quadratic singularity along the locus defined by the pair $\left\{p, p^{\prime}\right\}$. Associating to a local defining equation its hessian determines a natural isomorphism between $p^{*} \omega^{-1} \otimes p^{*} \omega^{-1}$ and the normal bundle of a divisor in the Deligne-Mumford boundary passing through $\overline{\mathcal{M}}(T)$.) We now see before us an algebro-geometric incarnation of the map that appears in the stability theorem: the set of normal vectors that point towards the interior $\overline{\mathcal{M}}_{g}$ is the restriction to $\mathcal{M}(S)$ of the total space of the direct sum of $\mathbb{C}^{\times}$bundles in this normal bundle. So $\mathcal{M}(S)_{Y}^{X}$ maps to the latter space, and although we do not have a morphism $\mathcal{M}(S)_{Y}^{X} \rightarrow \mathcal{M}_{g}^{n}$, the map on cohomology behaves as if it there were. In particular, the map $H^{\bullet}\left(\mathcal{M}_{g}^{n}\right) \rightarrow H^{\bullet}\left(\mathcal{M}(S)_{Y}^{X}\right)$ is a MHS morphism. So the stability theorem implies:

Theorem 4.1 (Algebro-geometric version of stability). Suppose that $X$ is contained in a connected component $S^{\prime}$ of $S$ of genus $g^{\prime}$, so that $\mathcal{M}\left(S^{\prime}\right)_{Y \cap S^{\prime}}^{X}$ appears as a factor of $\mathcal{M}(S)_{Y}^{X}$. Choose points in the remaining factors so that we have an inclusion of $\mathcal{M}\left(S^{\prime}\right)_{Y \cap S^{\prime}}^{X}$ in $\mathcal{M}(S)_{Y}^{X}$. Then for $k \leq c g^{\prime}$ the composite map

$$
H^{k}\left(\mathcal{M}_{g}^{n}\right) \rightarrow H^{k}\left(\mathcal{M}(S)_{Y}^{X}\right) \rightarrow H^{k}\left(\mathcal{M}\left(S^{\prime}\right)_{Y \cap S^{\prime}}^{X}\right)
$$

is an isomorphism and so is the map

$$
H^{k}\left(\mathcal{M}_{g^{\prime}}^{n}\right) \rightarrow H^{k}\left(\mathcal{M}\left(S^{\prime}\right)_{Y \cap S^{\prime}}^{X}\right)
$$

induced by the forgetful morphism $\mathcal{M}\left(S^{\prime}\right)_{Y \cap S^{\prime}}^{X} \rightarrow \mathcal{M}\left(S^{\prime}\right)^{X} \cong \mathcal{M}_{g^{\prime}}^{n}$. These maps are also MHS morphisms.

So the stable rational cohomology $H^{\bullet}\left(\Gamma_{\infty}^{n}\right)$ comes with a natural MHS. A geometric consequence of this result is that each stable rational cohomology class of $\mathcal{M}_{g}^{n}$ (that is, a class whose degree is in the stability range) extends across the open part of the blow up of $\overline{\mathcal{M}}(T)$ parameterizing the normal directions pointing towards the interior. Pikaart showed that these partial extensions can be made to come from a single extension to $\overline{\mathcal{M}}_{g}^{n}$, at least if $g$ is large compared with $k$. But then it is not hard to show that if this is possible for large $g$, then it is possible in the stable range and so the conclusion is:

Theorem 4.2 (Pikaart [73]). The restriction map $H^{k}\left(\overline{\mathcal{M}}_{g}^{n}\right) \rightarrow H^{k}\left(\mathcal{M}_{g}^{n}\right)$ is surjective in the stable range. Consequently, the $M H S$ on $H^{k}\left(\Gamma_{\infty}^{n}\right)$ is pure of weight $k$.

We illustrate this theorem with the known stable classes. We have seen in the previous section that $\overline{\mathcal{M}}_{g}^{n}$ comes with $n$ orbifold line bundles $x_{i}^{*} \omega, i=1, \ldots, n$. Let $\bar{\tau}_{i}$ denote the first Chern class of this line bundle, regarded as an element of $\mathrm{CH}^{1}\left(\overline{\mathcal{M}}_{g}^{n}\right)$. Denote its restriction to $\mathrm{CH}^{1}\left(\mathcal{M}_{g}^{n}\right)$ by $\tau_{i}$. (This notation should be regarded as refining our previous use of the $\tau_{i}$ 's, rather than being in conflict with it). As was noted before, the underlying cohomology class of $\tau_{i}$ is stable.

For the definition the tautological classes of $\mathcal{M}_{g}^{n}$, we shall not use Mumford's original definition, but a modification proposed by Arbarello-Cornalba. Consider the relatively dualizing sheaf on the universal curve over $\overline{\mathcal{M}}_{g}^{n}$ twisted by the $n$ sections $\omega\left(\left(x_{1}\right)+\cdots+\left(x_{n}\right)\right)$ and denote its first Chern class in $C H^{1}\left(\overline{\mathcal{M}}_{g}^{n}\right)$ by $\bar{\tau}$. 
Then the the $i$ th tautological class of $\overline{\mathcal{M}}_{g}^{n}$ is the direct image of $\bar{\tau}^{i+1} \in \mathrm{CH}^{i+1}\left(\overline{\mathcal{M}}_{g}^{1}\right)$ in $\mathrm{CH}^{i}\left(\overline{\mathcal{M}}_{g}\right)$. We denote this class by $\bar{\kappa}_{n, i}$ and its restriction to $\overline{\mathcal{M}}_{g}$ by $\kappa_{n, i}$ (but omit $n$ when it is zero). Note that $\bar{\kappa}_{n, 0}=2 g-2+n$. The cohomology class of $\kappa_{i}$ in $\mathcal{M}_{g}$ can be regarded as an element of $H^{2 i}\left(\Gamma_{g}\right)$ (of Hodge bidegree $(i, i)$ ); these cohomology classes stabilize and define the nonzero primitive element of degree $2 i$ alluded to in 2.2. We regard $\mathrm{CH}^{\bullet}\left(\overline{\mathcal{M}}_{g}^{n}\right)$ as a $\mathrm{CH}^{\bullet}\left(\overline{\mathcal{M}}_{g}\right)$ algebra via the obvious forgetful morphism, and view the classes $\bar{\kappa}_{i}$ as elements of $\mathrm{CH}^{\bullet}\left(\mathcal{M}_{g}^{n}\right)$ when appropriate. The relation between $\bar{\kappa}_{i}$ and $\bar{\kappa}_{n, i}$ is simply $\bar{\kappa}_{n, i}=\bar{\kappa}_{i}+\sum_{j=1}^{n}\left(\bar{\tau}_{j}\right)^{i}$ 沟.

As Arbarello-Cornalba point out in [4], the classes $\bar{\kappa}_{n, i}$ possess a nice property not enjoyed by Mumford's classes. First recall that every every stratum of $\overline{\mathcal{M}}_{g}^{n}$ is the image of a finite map $\overline{\mathcal{M}}(S)^{X \cup Y} \rightarrow \overline{\mathcal{M}}_{g}^{n}$ and that $\overline{\mathcal{M}}(S)^{X \cup Y}$ is a product of varieties of the type $\overline{\mathcal{M}}_{g_{\alpha}}^{n_{\alpha}}$. The pull-back of $\bar{\kappa}_{n, i}$ along this map is the sum of the classes $\bar{\kappa}_{n_{\alpha}, i}$ (pulled back along the projection $\mathrm{CH}^{\bullet}\left(\overline{\mathcal{M}}(S)^{X \cup Y}\right) \rightarrow \overline{\mathcal{M}}_{g_{\alpha}}^{n_{\alpha}}$ ). Since this behaviour is reminiscent of that of a primitive element in a Hopf algebra under the coproduct, we ask:

Question 4.3. Does this property characterize the collection $\left(\bar{\kappa}_{n, i}\right)_{n=0}^{\infty}$ up to a scalar factor?

4.1. Correspondences between moduli spaces. There is an altogether different way to relate the cohomology of the moduli spaces $\mathcal{M}_{g}^{n}$ for different values of $g$. This involves certain Hecke type correspondences. For simplicity we shall restrict ourselves to the undecorated case $n=0$. We return to the reference surface $S_{g}$ and suppose that we are given a subgroup $\pi$ of $\pi_{g}$ of finite index $d$, say. (But for what follows only its conjugacy class will matter.) This subgroup determines an unramified finite covering $\tilde{S} \rightarrow S$ of closed oriented surfaces. The genus $\tilde{g}$ of $\tilde{S}$ is then equal to $d(g-1)+1$. Consider the group of pairs $(\tilde{h}, h) \in \operatorname{Diff}^{+}(\tilde{S}) \times \operatorname{Diff}^{+}(S)$ such that $\tilde{h}$ is a lift of $h$. Let $\Gamma_{g}(\pi)$ be its group of connected components. The projection of $\Gamma_{g}(\pi) \rightarrow \Gamma_{g}$ has as kernel the group of covering transformations of $\tilde{S} \rightarrow S$ (so is finite) and its image consists of the outer automorphisms of $\pi_{g}$ that come from an automorphism which preserves the subgroup $\pi$ (so is of finite index, $e$, say). There is a corresponding finite covering of moduli spaces $p_{1}: \mathcal{M}_{g}(\pi) \rightarrow \mathcal{M}_{g}$, where $\mathcal{M}_{g}(\pi)$ is simply the coarse moduli space of finite unramified coverings of nonsingular complex projective curves $\tilde{C} \rightarrow C$ topologically equivalent to $\tilde{S} \rightarrow S$. There is also a finite map $p_{2}: \mathcal{M}_{g}(\pi) \rightarrow \mathcal{M}_{\tilde{g}}$. Together they define a one-to-finite correspondence $p_{2} p_{1}^{-1}$ from $\mathcal{M}_{g}$ to $\mathcal{M}_{\tilde{g}}$. This extends over the Deligne-Mumford compactifications: if $p_{1}: \overline{\mathcal{M}}_{g}(\pi) \rightarrow \overline{\mathcal{M}}_{g}$ denotes the normalization of $\overline{\mathcal{M}}_{g}$ in $\mathcal{M}_{g}(\pi)$, then $p_{2}$ extends to a finite morphism $p_{2}: \overline{\mathcal{M}}_{g}(\pi) \rightarrow \overline{\mathcal{M}}_{\tilde{g}}$. We have an induced map

$$
T_{\pi}:=e^{-1} p_{1 *} p_{2}^{*}: \mathrm{CH}^{\bullet}\left(\overline{\mathcal{M}}_{\tilde{g}}\right) \rightarrow \mathrm{CH}^{\bullet}\left(\overline{\mathcal{M}}_{g}\right)
$$

and likewise on cohomology. A computation shows that any monomial in the tautological classes is an "eigen class" for such correspondences:

Proposition 4.4. The map $T_{\pi}$ sends $\bar{\kappa}_{i_{1}} \bar{\kappa}_{i_{2}} \cdots \bar{\kappa}_{i_{r}}$ to $d^{r} \bar{\kappa}_{i_{1}} \bar{\kappa}_{i_{2}} \cdots \bar{\kappa}_{i_{r}}$.

This proposition suggests the consideration of, for given positive integers $r$ and $s$, sequences of classes $\left(x_{g} \in \mathrm{CH}^{s}\left(\overline{\mathcal{M}}_{g}\right)\right)_{g \geq 2}$ of fixed degree that have the property that $T_{\pi}\left(x_{g}\right)=d^{r} x_{(d-1) g+1}$ for each idex $d$ subgroup $\pi$ of $\pi_{g}$.

Question 4.5. Is for such a system the image of $x_{g}$ in $H^{\bullet}\left(\mathcal{M}_{g}\right)$ stable? Is it in fact a polynomial of degree $r$ in primitive stable classes? 
An affirmative answer would give us a notion of stability for the Chow groups of the moduli spaces $\mathcal{M}_{g}$.

\section{Chow Algebras and the Tautological Classes}

We have already encountered some of the basic classes on $\overline{\mathcal{M}}_{g}^{n}$ : the first Chern classes $\bar{\tau}_{i} \in \mathrm{CH}^{1}\left(\overline{\mathcal{M}}_{g}^{n}\right)(i=1, \ldots, n)$ and the tautological classes $\bar{\kappa}_{i}(i=1,2, \ldots)$. More such classes come from the boundary: if $\prod_{i} \overline{\mathcal{M}}_{g_{i}}^{n_{i}} \rightarrow \overline{\mathcal{M}}_{g}$ is a Galois covering of a stratum of the boundary as in Section 4, then we can add to these the pushforwards along this map of the exterior products of the corresponding classes on the factors. Let us call the subalgebra of $\mathrm{CH}^{\bullet}\left(\overline{\mathcal{M}}_{g}^{n}\right)$ generated by all these classes the tautological subalgebra and denote it by $\mathcal{R}^{\bullet}\left(\overline{\mathcal{M}}_{g}^{n}\right)$. The image of this algebra in $\mathrm{CH}^{\bullet}\left(\mathcal{M}_{g}^{n}\right)$ is denoted by $\mathcal{R}^{\bullet}\left(\mathcal{M}_{g}^{n}\right)$; it is generated by the classes $\kappa_{n, i}(i=1,2, \ldots)$ and $\tau_{i}(i=1, \ldots, n)$. It is possible that these classes generate the rational Chow ring of $\overline{\mathcal{M}}_{g}^{n}$ modulo homological equivalence, but this is of course unknown. In any case, these subalgebras are preserved under pull-back and push-forward along the natural maps that we have met so far.

The first computations were done by Mumford [69] who found a presentation of $\mathrm{CH}^{\bullet}\left(\overline{\mathcal{M}}_{2}\right)$. Subsequently Faber [13 calculated $\mathrm{CH}^{\bullet}\left(\overline{\mathcal{M}}_{2}^{1}\right), \mathrm{CH}^{\bullet}\left(\overline{\mathcal{M}}_{3}\right)$ and obtained partial results on $\mathrm{CH}^{\bullet}\left(\overline{\mathcal{M}}_{4}\right)$. In all these cases the tautological algebra is the whole Chow algebra.

This is also the case for $\overline{\mathcal{M}}_{0}^{n}$, whose Chow algebra was computed by Keel. This is a very remarkable algebra which appears in other contexts. Because of this, we describe it explicitly. We first introduce notation for the divisor classes on $\overline{\mathcal{M}}_{0}^{n}$. The boundary divisor $\overline{\mathcal{M}}_{0}^{n}-\mathcal{M}_{0}^{n}$ parameterizes all singular stable $n$ pointed rational curves. Its components correspond to the topological types of $n$ pointed stable rational curves with exactly one singular point. Such curves have exactly two irreducible components. By collecting the points $x_{i}$ 's lying on the same component, we obtain a partition $P$ of $\{1, \ldots, n\}$ into two subsets. The stability property implies that both members of $P$ have at least two elements. We denote the corresponding class in $\mathrm{CH}^{1}\left(\overline{\mathcal{M}}_{0}^{n}\right)$ by $D(P)$.

Theorem 5.1 (Keel $49 \mid$ ). The Chow algebra $\mathrm{CH}^{\bullet}\left(\overline{\mathcal{M}}_{0}^{n}\right)$ coincides with $H^{\bullet}\left(\overline{\mathcal{M}}_{0}^{n}\right)$ and, as a $\mathbb{Q}$ algebra, is generated by the $D(P)$ 's subject to the following relations:

(i) If $\{i, j, k\}$ are distinct integers in $\{1, \ldots, n\}$, then the sum of the $D(P)$ 's for which $P$ separates $i$ from $\{j, k\}$ is independent of $i$ (and equals $\bar{\tau}_{i}$ ).

(ii) $D(P) \cdot D\left(P^{\prime}\right)=0$ if $P$ and $P^{\prime}$ are independent in the sense that the partition they generate has four nonempty members.

The relations (ii) are geometrically obvious since the divisors $D(P)$ and $D\left(P^{\prime}\right)$ do not meet if $P$ and $P^{\prime}$ are independent. The additive relations (i) are not difficult to see either: if $C$ is a stable $n$ pointed rational curve, then a moment of thought shows that there is a unique morphism $z: C \rightarrow \mathbb{P}^{1}$ that is an isomorphism on one irreducible component, constant on the other irreducible components and is such that $z\left(x_{i}\right)=1, z\left(x_{j}\right)=0$ and $z\left(x_{k}\right)=\infty$. The differential $z^{-1} d z$ restricted to $x_{i}$ defines a section of $x_{i}^{*} \omega$. The image of $z^{-1} d z$ in $T_{x_{i}}^{*} C$ vanishes precisely when $z$ collapses the irreducible component containing $x_{i}$. 
In [59, Manin derives a formula for the Poincaré polynomial of $\overline{\mathcal{M}}_{0}^{n}$. Such a formula was independently found by Getzler [17] who also obtained the $\mathcal{S}_{n}$ equivariant Poincaré polynomial of $H^{\bullet}\left(\overline{\mathcal{M}}_{0}^{n}\right)$. That is, he determined the character of the $\mathcal{S}_{n}$ representations $H^{k}\left(\overline{\mathcal{M}}_{0}^{n}\right), k \geq 0$. Kaufmann [53] recently gave a formula for the intersection number of classes of strata of complementary dimension.

We now turn to the Chow and cohomology algebras of the moduli spaces $\mathcal{M}_{g}$. First we list some results about the Chow algebras.

$$
\begin{aligned}
& \mathrm{CH}^{\bullet}\left(\mathcal{M}_{1}^{n}\right)=\mathbb{Q} \text { for } n=1,2 \text { (folklore) } \\
& \mathrm{CH}^{\bullet}\left(\mathcal{M}_{2}\right)=\mathbb{Q}(\text { folklore) } \\
& \left.\mathrm{CH}^{\bullet}\left(\mathcal{M}_{2}^{1}\right)=\mathbb{Q}[\tau] /\left(\tau^{2}\right) \text { (Mumford }[69]\right) \\
& \mathrm{CH}^{\bullet}\left(\mathcal{M}_{3}\right)=\mathbb{Q}\left[\kappa_{1}\right] /\left(\kappa_{1}^{2}\right) \text { (Faber }[13), \\
& \left.\mathrm{CH}^{\bullet}\left(\mathcal{M}_{3}^{1}\right)=\mathbb{Q}\left[\kappa_{1}, \tau\right] /\left(\kappa_{1}^{2}, 4 \tau^{2}+\tau \kappa_{1}\right) \text { (Faber } 13\right), \\
& \left.\mathrm{CH}^{\bullet}\left(\mathcal{M}_{4}\right)=\mathbb{Q}\left[\kappa_{1}\right] /\left(\kappa_{1}^{3}\right) \text { (Faber }[13]\right), \\
& \mathrm{CH}^{\bullet}\left(\mathcal{M}_{5}\right)=\mathbb{Q}\left[\kappa_{1}\right] /\left(\kappa_{1}^{4}\right) \text { (Izadi }[40] \text { combined with Faber [14]). }
\end{aligned}
$$

The reason that such computations can be made is that, when $g$ and $n$ are both small, the moduli space $\mathcal{M}_{g}^{n}$ has a concrete description. For example, when $g=2$, each curve is hyperelliptic and therefore given by configuration of 6 points on the projective line. In the case $g=3$ a nonhyperelliptic curve is realized by its canonical system as a quartic curve in $\mathbb{P}^{2}$. The double cover of the projective plane along this curve is a Del Pezzo surface of degree 2, i.e., is obtained by blowing up 7 points in the plane in general position. Curves of genus 4 and 5 can be described as complete intersections of multidegrees $(2,3)$ (in $\left.\mathbb{P}^{3}\right)$ and $(2,2,2)$ (in $\left.\mathbb{P}^{4}\right)$, respectively.

5.1. The tautological algebra of $\mathcal{M}_{g}$ and Faber's Conjecture. On the basis of numerous calculations Faber made the following conjecture.

Conjecture 5.2 (Faber). The tautological algebra $\mathcal{R}^{\bullet}\left(\mathcal{M}_{g}\right)$ is a graded Frobenius algebra with socle in degree $g-2$. That is, $\operatorname{dim} \mathcal{R}^{g-2}\left(\mathcal{M}_{g}\right)=1$, and the intersection product defines a nondegenerate bilinear form $\mathcal{R}^{i}\left(\mathcal{M}_{g}\right) \times \mathcal{R}^{g-2-i}\left(\mathcal{M}_{g}\right) \rightarrow$ $\mathcal{R}^{g-2}\left(\mathcal{M}_{g}\right)(i=0, \ldots, g-2)$. Moreover, $\kappa_{1}$ has the Lefschetz property in $\mathcal{R} \bullet\left(\mathcal{M}_{g}\right)$ in the sense that multiplication by $\left(\kappa_{1}\right)^{g-2-2 i}$ maps $\mathcal{R}^{i}\left(\mathcal{M}_{g}\right)$ isomorphically onto $\mathcal{R}^{g-2-2 i}\left(\mathcal{M}_{g}\right)$ for $0 \leq i \leq \frac{1}{2}(g-2)$.

Since the conjecture was made, evidence for it has been growing. For example:

Theorem 5.3 (Looijenga [57]). The algebra $\mathcal{R} \bullet\left(\mathcal{M}_{g}\right)$ is trivial in degree $>g-2$ and $\mathcal{R}^{g-2}\left(\mathcal{M}_{g}\right)$ is generated by the class of the hyperelliptic locus (a closed irreducible variety of codimension $g-2$ ).

In particular $\kappa_{1}^{g-1}=0$. Since $\kappa_{1}$ is ample on $\mathcal{M}_{g}$, we recover a theorem of Diaz 11 which asserts that every complete subvariety of $\mathcal{M}_{g}$ must be of dim $\leq g-2$.

Actually, in [57] a stronger result is proven, which, among other things, implies that $\mathcal{R}^{k}\left(\mathcal{M}_{g}^{n}\right)=0$ for $k>g-2+n$. An induction argument then shows that $\mathcal{R}^{3 g-3+n}\left(\overline{\mathcal{M}}_{g}^{n}\right)$ is spanned by the classes of the zero dimensional strata. But zero dimensional strata can be connected by one dimensional strata and the one dimensional strata are all rational. This shows that $\mathcal{R}^{3 g-3+n}\left(\overline{\mathcal{M}}_{g}^{n}\right) \cong \mathbb{Q}$.

Faber recently proved that the tautological class $\kappa_{g-2}$ is nonzero. To describe his result, we find it convenient to introduce a compactly supported version of the tautological algebra: let $\mathcal{R}_{c}^{\bullet}\left(\mathcal{M}_{g}^{n}\right)$ be defined as the set of elements in $\mathcal{R} \bullet\left(\overline{\mathcal{M}}_{g}^{n}\right)$ that restrict trivially to the Deligne-Mumford boundary. This is a graded ideal in 
$\mathcal{R}^{\bullet}\left(\overline{\mathcal{M}}_{g}^{n}\right)$ and the intersection product defines a map

$$
\mathcal{R}^{\bullet}\left(\mathcal{M}_{g}^{n}\right) \times \mathcal{R}_{c}^{\bullet}\left(\mathcal{M}_{g}^{n}\right) \rightarrow \mathcal{R}_{c}^{\bullet}\left(\mathcal{M}_{g}^{n}\right)
$$

that makes $\mathcal{R}_{c}^{\bullet}\left(\mathcal{M}_{g}^{n}\right)$ a $\mathcal{R}^{\bullet}\left(\mathcal{M}_{g}^{n}\right)$-module. Notice that every complete subvariety of $\mathcal{M}_{g}$ of codimension $d$ whose class is in $\mathcal{R}^{\bullet}\left(\overline{\mathcal{M}}_{g}^{n}\right)$ defines a nonzero element of $\mathcal{R}_{c}^{d}\left(\mathcal{M}_{g}^{n}\right)$ (but it is by no means clear that such elements span $\mathcal{R}_{c}^{\bullet}\left(\mathcal{M}_{g}^{n}\right)$ ). A somewhat stronger form of the first part of Faber's Conjecture is:

Conjecture 5.4. The intersection pairings

$$
\mathcal{R}^{k}\left(\mathcal{M}_{g}\right) \times \mathcal{R}_{c}^{3 g-3-k}\left(\mathcal{M}_{g}\right) \rightarrow \mathcal{R}_{c}^{3 g-3}\left(\mathcal{M}_{g}\right) \cong \mathbb{Q}, \quad k=0,1,2, \ldots
$$

are perfect (Poincaré duality) and $\mathcal{R}_{c}^{\bullet}\left(\mathcal{M}_{g}\right)$ is a free $\mathcal{R} \bullet\left(\mathcal{M}_{g}\right)$ module of rank one.

Faber [14] finds a compactly supported class $I_{g}$ in $\mathcal{R}_{c}^{2 g-1}\left(\mathcal{M}_{g}\right)$ with $\kappa_{g-2} \cdot I_{g} \neq 0$. So $\mathcal{R}_{c}^{\bullet}\left(\mathcal{M}_{g}\right)$ should be the ideal generated by this element. Faber verified his conjecture for genera $\leq 15$ by writing down many relations in $\mathcal{R} \bullet\left(\mathcal{M}_{g}\right)$ (this evidently gives an upper bound) and using the nonvanishing of $\kappa_{g-2}$ (this gives a surprisingly strong lower bound).

A refined form of Conjecture 5.4 should include a suitable Lefschetz property.

Question 5.5. Does the tautological ring of $\overline{\mathcal{M}}_{g}^{n}$ satisfy Poincaré duality? Does it have the Lefschetz property with respect to $\bar{\kappa}_{n, 1}$ ? (It is known that $\bar{\kappa}_{n, 1}$ is ample 8.)

5.2. Cohomology of some moduli spaces. As may be expected, even less is known about the cohomology algebras. Here is an incomplete list of special results. In genus 0 we have that the Chow algebra of $\mathcal{M}_{0}^{n}$ maps isomorphically onto its rational cohomology algebra. The same is true for $\mathcal{M}_{1}^{1}, \mathcal{M}_{1}^{2}, \mathcal{M}_{2}$ and $\mathcal{M}_{2}^{1}$. Furthermore it is proved in [54] that

$$
H^{\bullet}\left(\mathcal{M}_{3}\right)=\mathrm{CH}^{\bullet}\left(\mathcal{M}_{3}\right)+\mathbb{Q} u,
$$

where $u$ is a class of degree 6 of Hodge bidegree $(6,6)$ and

$$
H^{\bullet}\left(\mathcal{M}_{3}^{1}\right)=\mathrm{CH}^{\bullet}\left(\mathcal{M}_{3}^{1}\right)+\mathbb{Q} u+\mathbb{Q} u \tau+\mathbb{Q} u \kappa_{1}+\mathbb{Q} v
$$

where $v$ is a class of degree 7 and of Hodge bidegree $(6,6)$

\section{The Ribbon Graph Picture}

Around 1981 Thurston, Mumford and Harer observed that partial completions of the Teichmüller spaces $\mathcal{X}_{g}^{n}$ with $n>0$ possess two natural $\Gamma_{g}^{n}$ equivariant triangulations. One is based on the hyperbolic geometry of $S_{g}^{n}$ (Thurston) and the other based on the singular euclidean geometry of $S_{g}^{n}$ (Mumford, Harer). The last approach was actually a direct, but very powerful application of work that Jenkins and Strebel had done 10-20 years earlier. It is this approach that we shall explain.

The basic notion is that of a ribbon graph. This is a finite graph $G$ together with a cyclic order on the set of oriented edges emanating from each vertex. As we shall see, there is a canonical construction of a surface that contains $G$ and of which $G$ is a deformation retract. This construction should explain the name. We

\footnotetext{
${ }^{3}$ For us a graph is a cell complex of pure dimension one; its zero cells are called vertices and its one cells edges. So it has no isolated vertices.

${ }^{4} \mathrm{An}$ oriented edge of a graph is an edge together with an orientation of it.
} 
first give a somewhat more abstract characterization of ribbon graphs which is very useful in some applications.

Let $X(G)$ be the set of oriented edges of $G$. Let $\sigma_{1}$ be the involution of $X(G)$ that reverses the orientation of each edge. The set $X_{1}(G)$ of $\sigma_{1}$ orbits can be identified with the set of edges of $G$. The cyclic orderings define another permutation $\sigma_{0}$ of $X(G)$ as follows. Each oriented edge $e$ has an initial vertex in $(e)$ and a terminal vertex term $(e)$. Define $\sigma_{0}(e)$ to be the successor of $e$ with respect to the given cyclic order on the set of oriented edges that have in $(e)$ as their initial vertex. The set of orbits $X_{0}(G)$ of $\sigma_{0}$ can be identified with the set of vertices of $G$. Put $\sigma_{\infty}:=\left(\sigma_{1} \sigma_{0}\right)^{-1}=\sigma_{0}^{-1} \sigma_{1}$. Call an orbit of this permutation a boundary cycle. (Draw a picture to see why.) The set of boundary cycles wil be denoted $X_{\infty}(G)$. These data form a complete invariant of $G$, for we can reverse the construction and associate to a finite nonempty set $X$ endowed with a fixed point free involution $\sigma_{1}$ and a permutation $\sigma_{0}$ of $X$, a ribbon graph $G\left(X, \sigma_{0}, \sigma_{1}\right)$ whose oriented edges are indexed by $X$ and such that $\sigma_{0}$ and $\sigma_{1}$ are the permutations defined above.

For every oriented edge $e$ of $G$ we form the one point compactification $\Delta_{e}$ of the half strip $e \times[0, \infty)$; this is just a 2 -simplex, parameterized in an unusual way. We make identifications along the boundaries of these simplices with the help of $\sigma_{0}$ and $\sigma_{\infty}: e \times\{0\}$ is identified with $\sigma_{1} e \times\{0\}$ and and $\{\operatorname{term}(e)\} \times[0, \infty)$ with $\left\{\operatorname{in}\left(\sigma_{\infty} e\right)\right\} \times[0, \infty)$ (in either case, the identification map is essentially the identity). This is easily seen to be compact triangulated surface that contains $G$ as a subcomplex. Its vertex set can be identified with the disjoint union of the vertex set of $G$ (so $X_{0}(G)$ ) and $X_{\infty}(G)$. We call vertices of the latter type cusps. Notice that $G$ is a deformation retract of $S(G)-X_{\infty}(G)$ and that the surface is canonically oriented if we insist that the cyclic orderings of the edges emanating from each vertex are induced by the orientation.

Suppose that we are given a metric $l$ on $G$. That is, a function that assigns to every (unoriented) edge of $X$ a positive real number. Give $[0, \infty)$ the standard metric and every half strip $e \times[0, \infty)$ the product metric. This defines (at least locally) a metric on $S(G)$. This metric is euclidean except possibly at the vertices. However, it is not difficult to show that the underlying conformal structure extends across all the vertices of $S(G)$ so that we end up with a compact Riemann surface $C(G, l)$. Notice that each cusp has a "circumference" - this is the length of the associated boundary cycle. It is clear that we get the same complex structure if $l$ is replaced by a positive multiple of it and so we may just as well assume that the total length of $G$ is 1 . With this convention, the sum of the circumferences of the cusps is 2 .

The work of Jenkins and Strebel shows that all compact Riemann surfaces arise in this way:

Theorem 6.1 (Strebel 76]). Suppose that $C$ is a compact connected Riemann surface and that $P$ is a finite subset of $C$ such that $C-P$ has negative euler characteristic. Then for every function $\lambda: P \rightarrow[0, \infty)$ that is not identically zero there exists a metrized ribbon graph $(G, l)$ and an isomorphism $h: C \stackrel{\sim}{\rightarrow} C(G, l)$ of Riemann surfaces such that

(i) $h(P)$ is contained in the vertex set of $S(G)$;

(ii) $h(P)$ contains every vertex of $G$ of degree $\leq 2$ and every cusp;

(iii) if $\lambda(p)=0$, then $h(p)$ is a vertex of $G$, and if $\lambda(p)>0$, then $h(p)$ is a cusp of $G$ of circumference $\frac{1}{2} \lambda(p)$. 
Moreover, $(G, l, h)$ is unique up to the obvious notion of isomorphism.

The results of Strebel also include a continuity property: a continuous variation of the complex structure on $C$ corresponds to a continuous variation of $(G, l)$ in a sense that we make precise. Denote by $\mathcal{R G}_{g}^{n}$ the set of isomorphism classes of ribbon graphs $G$ that are marked in the sense that we are given an isotopy class of homeomorphisms $h: S_{g} \rightarrow S(G)$ whose restriction to $\left\{x_{1}, \ldots, x_{n}\right\}$ satisfies properties (i) and (ii) of the above theorem. On this set $\Gamma_{g}^{n}$ acts, and it is easy to see that the number of orbits of markings is finite.

Suppose that $(G,[h])$ represents an element of $\mathcal{R G}_{g}^{n}$. Denote the geometric realization of the abstract simplex on the set $X_{1}(G)$ by $\Delta(G)$. Notice that the metrics $l$ on $G$ that give $G$ unit length are parameterized by the interior of $\Delta(G)$. The circumference function $\lambda$ extends to a simplicial projection of $\Delta(G)$ to the standard $(n-1)$-simplex $\Delta^{n-1}$.

Let $s$ be an edge of $G$ that is not a loop and does not connect two special vertices. Then collapsing that edge yields a member $(G / s,[h] / s)$ of $\mathcal{R G}_{g}^{n}$. We can regard $\Delta(G / s)$ as a face of $\Delta(G)$. Making these identifications produces a simplicial complex which we will denote by $\widehat{\mathbb{X}}_{g}^{n}$. The circumferences of the cusps define a simplicial map $\widehat{\mathbb{X}}_{g}^{n} \rightarrow \Delta^{n-1}$. We have a simplicial action of $\Gamma_{g}^{n}$ on $\widehat{\mathbb{X}}_{g}^{n}$ which preserves the fibers of $\lambda$. The union of relative interiors of simplices of $\widehat{\mathbb{X}}_{g}^{n}$ indexed by the elements of $\mathcal{R} \mathcal{G}_{g}^{n}$ is an open subset $\mathbb{X}_{g}^{n}$ of $\widehat{\mathbb{X}}_{g}^{n}$. The results of Strebel can be strengthened to:

Proposition 6.2 (cf. [56]). The above construction defines a $\Gamma_{g}^{n}$ equivariant homeomorphism of $\mathbb{X}_{g}^{n}$ onto $\mathcal{X}_{g}^{n} \times \Delta^{n-1}$.

Now consider the quotient space

$$
\widehat{\mathbb{M}}_{g}^{n}:=\Gamma_{g}^{n} \backslash \widehat{\mathbb{X}}_{g}^{n}
$$

This is a finite simplicial orbicomplex that is equipped with a simplicial map $\lambda: \widehat{\mathbb{M}}_{g}^{n} \rightarrow \Delta^{n-1}$. We regard this complex as a compactification of its open subset $\mathbb{M}_{g}^{n}:=\Gamma_{g}^{n} \backslash \mathbb{X}_{g}^{n}$. According to the above theorem, the latter is canonically homeomorphic with $\mathcal{M}_{g}^{n} \times \Delta^{n-1}$. This raises the question of how this compactification compares to that of Deligne-Mumford. The answer is essentially due to Kontsevich:

Theorem 6.3 (Kontsevich [50], see also [56]). The simplicial orbicomplex $\widehat{\mathbb{M}}_{g}^{n}$ is a quotient space of $\overline{\mathcal{M}}_{g}^{n} \times \Delta^{n-1}$. Moreover, the part of $\widehat{\mathbb{M}}_{g}^{n}$ where $\lambda_{i}>0$ carries an oriented piecewise linear circle bundle (in the orbifold sense) whose pull-back to $\overline{\mathcal{M}}_{g}^{n} \times\left\{\lambda \in \Delta^{n-1} \mid \lambda_{i}>0\right\}$ is the oriented circle bundle coming from the standard line bundle $\bar{\tau}_{i}$. In particular, the part of $\widehat{\mathbb{M}}_{g}^{n}$ lying over the interior of $\Delta^{n-1}$ carries the tautological classes $\bar{\tau}_{i}, i=1, \ldots, n$.

The defining equivalence relation on $\overline{\mathcal{M}}_{g}^{n} \times \Delta^{n-1}$ is a little subtle and we refer to [56 for details regarding both statement and proof. []

This compactification of $\mathcal{M}_{g}^{n} \times \Delta^{n-1}$ plays a crucial rôle in Kontsevich's proof of the Witten conjectures. There are however earlier applications. These include Harer's stability theorem we met before, the computation of the Euler characteristic

\footnotetext{
${ }^{5}$ The space used by Kontsevich is not quite $\widehat{\mathbb{M}}_{g}^{n}$, but basically the part lying over the interior of $\Delta^{n-1}$ times a half line. In this case the circumference map $\lambda$ has image $(0, \infty)^{n}$.
} 
of $\mathcal{M}_{g}^{n}$, and the proof that that $\Gamma_{g}^{n}$ is a virtual duality group of dimension $4 g-4+n$. We shall not explain the relation with stability here, but we will briefly touch on the other applications.

There is also a remarkable arithmetic aspect of ribbon graphs that is presently under intense investigation, but that we merely mention in passing. This is the observation, made by Grothendieck in a research proposal [20], that for a metrized ribbon graph $(G, l)$ all of whose edges have equal length, the corresponding Riemann surface $C(G, l)$ is, in a canonical way, a ramified covering of the Riemann sphere $\mathbb{P}^{1}$ with ramification locus contained in $\{0,1, \infty\}$. The graph $G$ appears here as the preimage of the interval $[0,1]$, its vertex set as the preimage of 0 and the set of cusps as the preimage of $\infty$. The preimage of 1 consists of the midpoints of the edges. At these points we have simple ramification. A covering of this type is naturally an algebraic curve defined over some number field. Conversely, every connected covering of the Riemann sphere of this type arises in this manner. The absolute Galois group of $\mathbb{Q}$ acts on the collection of isomorphism types of such coverings, and thus also on each finite set $\mathcal{G}_{g}^{n}$. It is very difficult to come to grips with this action. For more information we refer to the collection [21] and to Grothendieck's manuscripts [19] and 20]. Since these metrized ribbon graphs represent the barycenters of the simplices of $\mathbb{M}_{g}^{n}$, one can also think of this as an action of the absolute Galois group on the simplices of $\mathbb{M}_{g}^{n}$, but it is not clear to us what the significance of that fact is.

6.1. Virtual duality and virtual Euler characteristic. We first make some observations about simplicial complexes. Let $K$ be a simplicial complex, $L$ a subcomplex. Set $U:=K-L$. Then $U$ admits as a canonical deformation retract onto the union of the closed simplices of the barycentric barycentric subdivision of $K$ that lie in $U$. This is a subcomplex, called the spine of $U$, whose $k$-simplices correspond to strictly increasing chains $\sigma_{0} \subset \sigma_{1} \subset \cdots \subset \sigma_{k}$ of simplices of $K$ not in $L$. Further, if $\Gamma$ is a group of automorphisms of $K$ that preserves $L$, and if

(i) $U$ is a contractible,

(ii) a subgroup of $\Gamma$ of finite index acts freely on $U$,

then $\Gamma \backslash U$ is a simplicial 'orbicomplex' that is also a classifying space for $\Gamma$. It has $\Gamma \backslash \operatorname{spine}(U)$ as deformation retract and so the dimension of this spine is an upper bound for the virtual homological dimension of $\Gamma$.

We apply this in the situation where $K$ is the preimage of the first vertex of $\Delta^{n-1}$ in $\widehat{\mathbb{X}}_{g}^{n}$ under $\lambda$ and $U=K \cap \mathbb{X}_{g}^{n}$. Notice that $U \cong \mathcal{X}_{g}^{n}$. The simplices meeting $U$ are indexed by the elements of $\mathcal{R \mathcal { G }}_{g}^{n}$ with a single boundary cycle. A simple calculation shows that the number of edges of such a graph is at most $6 g-5+2 n$ and at least $2 g-1+n$. So the spine of $U$ has dimension $\leq 4 g-4+n$. One can verify that this is, in fact, an equality. It follows that $U$ admits a subcomplex of dimension $4 g-4+n$ as an equivariant deformation retract. Hence:

Theorem 6.4 (Harer 28). If $n \geq 1$, then for every level structure, the moduli space $\mathcal{M}_{g}^{n}[G]$ contains a subcomplex of dimension $4 g-4+n$ as a deformation retract.

Problem 6.5. Is there a Lefschetz type of proof of this fact?

Let us return to the general situation considered earlier and suppose, in addition, that 
(iii) $\Gamma \backslash K$ is finite complex, and

(iv) $U$ is a simplicial manifold of dimension $d$, say.

These two conditions that are satisfied in the case at hand. It is then natural (and standard) to assign to each simplex of $K$ the weight that is the reciprocal of the order of its $\Gamma$ stabilizer. This weighting is constant on orbits. Wall's Euler characteristic of $\Gamma$ is simply the usual alternating sum of the number of $\Gamma$ orbits of simplices not in $L$, except that each is counted with its weight. Equivalently, it is the orbifold euler characteristic of the quotient $\Gamma \backslash K$.

In the present case, a ribbon graph $G$ defining a member of $\mathcal{R} \mathcal{G}_{g}^{n}$ gives a contribution $|\operatorname{Aut}(G)|^{-1}(-1)^{\left|X_{1}(G)\right|}$ to the virtual Euler characteristic. The computation of the resulting sum is a combinatorial problem that was first solved by Harer and Zagier. Kontsevich [50] later gave a shorter proof. The answer is:

Theorem 6.6 (Harer-Zagier [34]). The orbifold Euler characteristic of $\mathcal{M}_{g}^{n}$ equals

$$
\frac{1}{2-2 g} \zeta(1-2 g) \text { if } n=0 \text {, and }(-1)^{n-1} \frac{(2 g+n-3) !}{(2 g-2) !} \zeta(1-2 g) \text { if } n>0 .
$$

Here $\zeta$ denotes the Riemann zeta function.

Harer and Zagier also find formulae for the actual Euler characteristics of $\mathcal{M}_{g}^{1}$ and $\mathcal{M}_{g}$. These are often negative so that there must be lot of cohomology in odd degrees.

For the discussion of virtual duality we go back to the general situation and assume that beyond the four conditions already imposed we have

(v) $L$ has the homotopy type of a bouquet of $r$-spheres.

Then the theory of Bieri-Eckmann can be invoked in a virtual setting: if we set $D:=\tilde{H}_{r}(L ; \mathbb{Z})$ and regard $D$ as a $\Gamma$ module in an obvious way, then $H_{d-r-1}(\Gamma, D)$ is of rank one and for any $\Gamma$-module $V$ with rational coefficients the cap products

$$
\cap: H^{k}(\Gamma ; V) \otimes H_{d-r-1}(\Gamma ; D) \rightarrow H_{d-r-1-k}(\Gamma ; V \otimes D), \quad k=0,1,2, \ldots
$$

are isomorphisms. One calls $D$ the Steinberg module of $\Gamma$.

Harer [28] proves that in the present case hypothesis (v) is satisfied: $L$ is a subcomplex of dimension $2 g-3-n$ which is $(2 g-4-n)$-connected.

We shall call the corresponding orbifold local system $\mathbb{D}$ over $\mathcal{M}_{g}^{n}$ the Steinberg sheaf. The homology group $H_{4 g-4+n}\left(\mathcal{M}_{g}^{n} ; \mathbb{D}\right)$ is of rank one. For every orbifold local system $\mathbb{V}$ of rational vector spaces on $\mathcal{M}_{g}^{n}$, cap product with a generator of this homology group defines isomorphisms

$$
H^{k}\left(\mathcal{M}_{g}^{n} ; \mathbb{V}\right) \stackrel{\sim}{\rightarrow} H_{4 g-4+n-k}\left(\mathcal{M}_{g}^{n} ; \mathbb{V} \otimes \mathbb{D}\right), \quad k=0,1,2, \ldots
$$

In particular, taking $\mathbb{V}$ to be $\mathbb{Q}$, we see that $H_{\bullet}\left(\mathcal{M}_{g}^{n} ; \mathbb{D}\right)$ has a canonical MHS. This suggests that $\mathbb{D}$ has some Hodge theoretic significance. Unfortunately it is not of finite rank, yet we wonder:

Question 6.7. Is the Steinberg sheaf motivic? In particular, does it have natural completions that carry (compatible) Hodge and étale structures?

6.2. Intersection numbers on the Deligne-Mumford completion. The intersection numbers in question are those defined by monomials in the $\bar{\tau}_{i}$ 's. To be precise, define for every such monomial $\bar{\tau}_{1}^{d_{1}}, \ldots, \bar{\tau}_{n}^{d_{n}}$ (with all $d_{i}>0$ ) the intersection number $\int_{\mathcal{M}_{g}^{n}} \bar{\tau}_{1}^{d_{1}}, \ldots, \bar{\tau}_{n}^{d_{n}}$ where $g$ is chosen in such a way that this has a 
possibility of being nonzero: $3 g-3+n=d_{1}+\cdots+d_{n}$. A physics interpretation suggests that we combine these numbers into the generating function

$$
\sum_{n=1}^{\infty} \frac{1}{n !} \sum_{g>1-\frac{1}{2} n} \sum_{d_{1}+\cdots+d_{n}=3 g-3+n} t_{d_{1}} \ldots t_{d_{n}} \int_{\mathcal{M}_{g}^{n}} \bar{\tau}_{1}^{d_{1}} \ldots \bar{\tau}_{n}^{d_{n}} .
$$

Now pass to a new set of variables $T_{1}, T_{3}, T_{5}, \ldots$ by setting $t_{i}=1.3 .5 . \cdots(2 i+$ 1) $T_{2 i+1}$. The resulting expansion $F\left(T_{1}, T_{3}, T_{5}, \ldots\right)$ encodes all these intersection numbers.

Witten 77 conjectured two other characterizations of this function both of which allow computation of its coefficients. These were proved by Kontsevich in his celebrated paper [50. Perhaps the most useful characterization is the one which says that $F$ is killed by a Lie algebra of differential operators isomorphic to the Lie algebra of polynomial vector fields in one variable. This Lie algebra comes with a basis $\left(L_{k}\right)_{k \geq-1}$ corresponding to the vector fields $\left(z^{k} \partial / \partial z\right)_{k>-1}$ and Witten verified the identities $L_{k}(F)=0$ for $k=-1,0$ within the realm of algebraic geometry. However no such proof is known for $k \geq 1$. Kontsevich's strategy is to represent the classes $\bar{\tau}_{1}^{d_{1}} \cdots \bar{\tau}_{n}^{d_{n}}$ by piecewise differential forms on on the ribbon graph model that can actually be integrated. This allows him to convert the intersection numbers into weighted sums over ribbon graphs. This leads to a new characterization of the generating function that is more manageable. Still a great deal of ingenuity is needed to complete the proof of Witten's Conjecture.

\section{Torelli Groups ANd Moduli}

In the early 80s, Dennis Johnson published a series of pioneering papers 42 , 43, 44 on the Torelli groups. Although this work is in geometric topology, it has several interesting applications to algebraic geometry. Here we review some of his work.

First a remark on notation. In the remainder of the paper we will write $V_{g}$ for the symplectic vector space $H_{1}\left(S_{g}\right)$ and $S p_{g}(\mathbb{Z})$ for the group $\operatorname{Aut}\left(H_{1}\left(S_{g}, \mathbb{Z}\right),\langle\rangle,\right)$; this does not really clash with standard notation, since a choice of a symplectic basis of $H_{1}\left(S_{g}, \mathbb{Z}\right)$ identifies this with the standard integral symplectic group of genus $g$. Likewise, $S p_{g}$ will stand for the algebraic $\mathbb{Q}$-group defined by the symplectic transformations in $V_{g}$; so its group of $\mathbb{Q}$-points, $S p_{g}(\mathbb{Q})$, is just the group of symplectic automorphisms of $V_{g}$.

The mapping class group $\Gamma_{g, r}^{n}$ acts on the homology of the reference surface $S_{g}$. Since each of its elements preserves the orientation of $S_{g}$, we have a homomorphism

$$
\Gamma_{g, r}^{n} \rightarrow S p_{g}(\mathbb{Z}) .
$$

which is surjective. The Torelli group $T_{g, r}^{n}$ is defined to be its kernel $]^{\text {f }}$ so that we have an extension

$$
1 \rightarrow T_{g, r}^{n} \rightarrow \Gamma_{g, r}^{n} \rightarrow S p_{g}(\mathbb{Z}) \rightarrow 1 .
$$

The homology groups of $T_{g, r}^{n}$ are therefore $S p_{g}(\mathbb{Z})$ modules.

The simplest kind of element of $T_{g, r}^{n}$ is a Dehn twist along a simple loop in $S_{g}^{n+r}$ that separates $S$ into two connected components. We call such a loop a separating simple loop. Another type of element of $T_{g, r}^{n}$ is determined by a separating pair of simple loops. This is a pair of two disjoint nonisotopic loops $\alpha_{1}, \alpha_{2}$ on $S_{g}^{n+r}$

${ }^{6}$ Note that there is no general agreement on the definition of $T_{g, r}^{n}$ when $r+n>1$. 
that together separate $S$ into two connected components. The Dehn twist along $\alpha_{1}$ composed with the inverse of the Dehn twist along $\alpha_{2}$ is in $T_{g, r}^{n}$. The first of Johnson's results is:

Theorem 7.1 (Johnson 42, 43, 44). When $g \geq 3, T_{g, r}^{n}$ is generated by elements associated to a finite number of separating simple loops and a finite number of separating pairs of simple loops. If $\left[S_{g}\right] \in \wedge^{2} H_{1}\left(S_{g}, \mathbb{Z}\right)$ corresponds to the fundamental class of $S_{g}$, then there are natural $S p_{g}(\mathbb{Z})$ equivariant surjective homomorphisms

$$
\tau_{g}^{1}: T_{g}^{1} \rightarrow \wedge^{3} H_{1}\left(S_{g}, \mathbb{Z}\right) \text { and } \tau_{g}: T_{g} \rightarrow \wedge^{3} H_{1}\left(S_{g}, \mathbb{Z}\right) /\left(\left[S_{g}\right] \wedge H_{1}\left(S_{g}, \mathbb{Z}\right)\right) .
$$

In both cases, the kernel of $\tau$ is the subgroup generated by the elements associated to simple separating loops. Finally, the kernels of the induced homomorphisms

$$
H_{1}\left(T_{g}^{1} ; \mathbb{Z}\right) \rightarrow \wedge^{3} H_{1}\left(S_{g}, \mathbb{Z}\right) \text { and } H_{1}\left(T_{g}^{1} ; \mathbb{Z}\right) \rightarrow \wedge^{3} H_{1}\left(S_{g}, \mathbb{Z}\right) /\left(\left[S_{g}\right] \wedge H_{1}\left(S_{g}, \mathbb{Z}\right)\right)
$$

are both 2-torsion.

Johnson also finds an explicit description of this 2-torsion. We will give it in a moment, but first we want to point out an algebro-geometric consequence of this theorem. Let $\widetilde{\mathcal{M}}_{g} \subset \overline{\mathcal{M}}_{g}$ be the complement of the irreducible divisor whose generic point parametrizes irreducible singular stable curves, and let $\widetilde{\mathcal{M}}_{g}^{1}$ be its preimage in $\overline{\mathcal{M}}_{g}^{1}$.

Corollary 7.2. When $g \geq 3$, the orbifold fundamental group of $\widetilde{\mathcal{M}}_{g}$ (resp. $\widetilde{\mathcal{M}}_{g}^{1}$ ) is isomorphic to an extension of $S p_{g}(\mathbb{Z})$ by $\wedge^{3} H_{1}\left(S_{g}, \mathbb{Z}\right) /\left(\left[S_{g}\right] \wedge H_{1}\left(S_{g}, \mathbb{Z}\right)\right.$ ) (resp. $\left.\wedge^{3} H_{1}\left(S_{g}, \mathbb{Z}\right)\right)$.

Johnson's theorem shows that the $S p_{g}(\mathbb{Z})$ action on $H_{1}\left(T_{g, r}^{n}\right)$ is the restriction of a representation of the algebraic group $S p_{g}$. We shall see shortly the importance of this property. Let $\lambda_{1}, \lambda_{2}, \ldots, \lambda_{g}$ be a fundamental set of weights of $S p_{g}$ so that $\lambda_{j}$ corresponds to the $j$ th fundamental representation of $S p_{g}$. This last representation can be realized as the natural $S p_{g}$ action on the primitive part of $\wedge^{j} V_{g}$.

The next result follows from Johnson's Theorem by standard arguments.

Corollary 7.3. For each $g \geq 3$, there is a natural $S p_{g}(\mathbb{Z})$ equivariant isomorphism

$$
\tau_{g, r}^{n}: H^{1}\left(T_{g, r}^{n}\right) \stackrel{\sim}{\rightarrow} V\left(\lambda_{3}\right) \oplus V\left(\lambda_{1}\right)^{\oplus(r+n)} .
$$

A theorem of Ragunathan [75] implies that every finite index subgroup of $S p_{g}(\mathbb{Z})$ has zero first Betti number when $g \geq 2$. So Johnson's computation also gives:

Corollary 7.4. If $g \geq 3$, then every finite index subgroup of $\Gamma_{g, r}^{n}$ that contains $T_{g, r}^{n}$ has zero first Betti number.

The situation is very different when $g<3$. The Torelli group $T_{1}$ is the trivial group, while Geoff Mess 60] proved that when $g=2, T_{2}$ is a countably generated free group. He also computed $H_{1}\left(T_{2}, \mathbb{Z}\right)$. It is the $S p_{2}(\mathbb{Z})$ module obtained by inducing the trivial representation up to $S p_{2}(\mathbb{Z})$ from the stabilizer $(\mathbb{Z} / 2) \ltimes\left(S L_{2}(\mathbb{Z}) \times S L_{2}(\mathbb{Z})\right)$ of a decomposition of $H_{1}\left(S_{2}, \mathbb{Z}\right)$ into two symplectic modules each of rank 2 . (We shall sketch a proof in the next subsection.) It is still unknown whether, for any $g \geq 3, T_{g}$ is finitely presented.

Problem 7.5. Determine whether $T_{g}$ is finitely presented when $g$ is sufficiently large. 
Next, we describe Johnson's computation of the torsion in $H_{1}\left(T_{g}, \mathbb{Z}\right)$. Denote the field of two elements by $\mathbb{F}_{2}$. Recall that an $\mathbb{F}_{2}$ quadratic form on $H_{1}\left(S_{g} ; \mathbb{F}_{2}\right)$ associated to the mod two symplectic form $\langle$,$\rangle on H_{1}\left(S_{g} ; \mathbb{F}_{2}\right)$ is a function $\omega$ : $H_{1}\left(S_{g} ; \mathbb{F}_{2}\right) \rightarrow \mathbb{F}_{2}$ satisfying

$$
\omega(a+b)=\omega(a)+\omega(b)+\langle a, b\rangle .
$$

The difference between any two such is an element of $H^{1}\left(S_{g}, \mathbb{F}_{2}\right)$. This makes the set $\Omega_{g}$ of such quadratic forms an affine space over the $\mathbb{F}_{2}$ vector space $H^{1}\left(S_{g} ; \mathbb{F}_{2}\right)$. Denote the algebra of $\mathbb{F}_{2}$ valued functions on $\Omega_{g}$ by $S \Omega_{g}$. All such functions are given by polynomials and so we have a filtration

$$
\mathbb{F}_{2}=S_{0} \Omega_{g} \subset S_{1} \Omega_{g} \subset S_{2} \Omega_{g} \subset \cdots \subset S \Omega_{g},
$$

where $S^{d} \Omega_{g}$ denote the space of polynomial functions of degree $\leq d$. Since $f=f^{2}$ for each $f \in S \Omega_{g}$, the associated graded algebra is naturally isomorphic to the exterior algebra $\wedge^{\bullet} H_{1}\left(S_{g} ; \mathbb{F}_{2}\right)$. The algebra $S \Omega_{g}$ has as a distinguished element which is called the Arf invariant, denoted here by arf. If $a_{1}, \ldots, a_{g}, b_{1}, \ldots, b_{g}$ is a symplectic basis of $H^{1}\left(S ; \mathbb{F}_{2}\right)$, then arf is defined by

$$
\text { arf : } \omega \mapsto \sum_{i} \omega\left(a_{i}\right) \omega\left(b_{i}\right) \text {. }
$$

It is an element of $S_{2} \Omega_{g}$, and its zero set $\Psi_{g}$ is an affine quadric in $\Omega_{g}$. Let $S_{d} \Psi_{g}$ denote the image of $S_{d} \Omega_{g}$ in the set of $\mathbb{F}_{2}$ valued functions on $\Psi_{g}$.

Theorem 7.6 (Johnson 44]). There are natural $S p_{g}\left(\mathbb{F}_{2}\right)$ equivariant isomorphisms

$$
\begin{gathered}
\sigma_{g, 1}: H_{1}\left(T_{g, 1} ; \mathbb{F}_{2}\right) \stackrel{\sim}{\rightarrow} S_{3} \Omega_{g}, \quad \sigma_{g}^{1}: H_{1}\left(T_{g}^{1} ; \mathbb{F}_{2}\right) \stackrel{\sim}{\rightarrow} S_{3} \Omega_{g} / \mathbb{F}_{2} \text { arf }, \\
\sigma_{g}: H_{1}\left(T_{g} ; \mathbb{F}_{2}\right) \stackrel{\sim}{\rightarrow} S_{3} \Psi_{g} .
\end{gathered}
$$

These induce natural isomorphisms

$$
H_{1}\left(T_{g, 1} ; \mathbb{Z}\right)_{\text {tor }} \cong S_{2} \Omega_{g}, \quad H_{1}\left(T_{g}^{1} ; \mathbb{Z}\right)_{\text {tor }} \cong H_{1}\left(T_{g} ; \mathbb{Z}\right)_{\text {tor }} \cong S_{2} \Psi_{g}
$$

Moreover, the natural isomorphisms

$$
\phi_{g, r}^{n}: H_{1}\left(T_{g, r}^{n} ; \mathbb{F}_{2}\right) / H_{1}\left(T_{g, r}^{n} ; \mathbb{Z}\right)_{\text {tor }} \stackrel{\sim}{\rightarrow}\left[H_{1}\left(T_{g, r}^{n} ; \mathbb{Z}\right) / \text { torsion }\right] \otimes \mathbb{F}_{2}
$$

correspond, under the isomorphisms $\sigma_{g, r}^{n}$ and $\tau_{g, r}^{n}$, to the obvious isomorphisms

$$
\begin{gathered}
\phi_{g, 1}: S_{3} \Omega_{g} / S_{2} \Omega_{g} \stackrel{\sim}{\rightarrow} \wedge^{3} H_{1}\left(S_{g} ; \mathbb{F}_{2}\right), \quad \phi_{g}^{1}: S_{3} \Omega_{g} /\left(\mathbb{F}_{2} \text { arf }+S_{2} \Omega_{g}\right) \stackrel{\sim}{\rightarrow} \wedge^{3} H_{1}\left(S_{g} ; \mathbb{F}_{2}\right), \\
\phi_{g}: S_{3} \Psi_{g} / S_{2} \Psi_{g} \stackrel{\sim}{\rightarrow} \wedge^{3} H_{1}\left(S_{g} ; \mathbb{F}_{2}\right) /\left(\left[S_{g}\right] \wedge H_{1}\left(S_{g} ; \mathbb{F}_{2}\right)\right) .
\end{gathered}
$$

The homomorphisms $\tau_{g}^{1}$ and $\tau_{g}$ admit direct conceptual definitions that we will give later. Here we give a formula for the image of the standard generators of $T_{g, 1}$ in $\wedge^{3} H_{1}\left(S_{g} ; \mathbb{Z}\right)$ and in $S_{3} \Omega_{g}$ under $\tau_{g, 1}$ and $\sigma_{g, 1}$, respectively.

Let $\left(\alpha_{1}, \alpha_{2}\right)$ be a separating pair of simple loops. Let $t$ be the corresponding element of $T_{g, 1}$. The two loops decompose $S_{g}$ into two pieces $S^{\prime}$ and $S^{\prime \prime}$, say, where we suppose that $S^{\prime}$ contains the point $x_{1}$. We orient $\alpha_{1}$ and $\alpha_{2}$ as boundary components of $S^{\prime \prime}$. The resulting cycles define the same class $[\alpha]$ in $H_{1}\left(S^{\prime \prime} ; \mathbb{Z}\right)$ and this class spans the radical of the intersection pairing on $H_{1}\left(S^{\prime \prime} ; \mathbb{Z}\right)$. So there is a welldefined element in $\wedge^{2} H_{1}\left(S^{\prime \prime} ; \mathbb{Z}\right) /[\alpha] \wedge H_{1}\left(S^{\prime \prime} ; \mathbb{Z}\right)$ representing the intersection pairing on $H_{1}\left(S^{\prime \prime} ; \mathbb{Z}\right)$. Its wedge with $[\alpha]$ can be regarded as an element of $\wedge^{3} H_{1}\left(S^{\prime \prime} ; \mathbb{Z}\right)$. Since the inclusion $S^{\prime \prime} \subset S_{g}$ induces an injection on first homology, we can also view the latter as an element of $\wedge^{3} H_{1}\left(S_{g} ; \mathbb{Z}\right)$. This is the element $\tau_{g, 1}(t)$; it is clear that it only depends on the image of $t$ in $T_{g}^{1}$. 
Next we associate to $t$ a function $\sigma_{t}: \Omega_{g} \rightarrow \mathbb{F}_{2}$ as follows. If $\omega \in \Omega_{g}$ takes the value 1 on $[\alpha]$, then we put $\sigma_{t}(\omega)=0$; if it takes the value 0 on $[\alpha]$, then the restriction of $\omega$ to $H_{1}\left(S^{\prime \prime} ; \mathbb{F}_{2}\right)$ factors through a nondegenerate quadratic function on $H_{1}\left(S^{\prime \prime} ; \mathbb{F}_{2}\right) / \mathbb{F}_{2}[\alpha]$. Then $\sigma_{g, 1}(t)(\omega)$ is its Arf invariant. It can be shown that $\sigma_{g, 1}(t)$ lies in $S_{3} \Omega$.

Now suppose that $t$ is the element of $T_{g, 1}$ associated to a separating simple loop $\alpha$. Denote the pieces $S^{\prime}$ and $S^{\prime \prime}$ as before. In this case, $\tau_{g, 1}(t)$ is trivial and $\sigma_{g, 1}(t)$ is the element of $S \Omega_{g}$ that assigns to $\omega$ the Arf invariant of its restriction to $H_{1}\left(S^{\prime \prime}, \mathbb{F}_{2}\right)$. Notice that if $\alpha$ is a simple loop around $x_{1}$, then $\sigma_{g, 1}(t)$ is just the function arf. (This explains why we mod out by this function when passing from $T_{g, 1}$ to $T_{g}^{1}$.)

Without a base point there would be no way of telling $S^{\prime}$ and $S^{\prime \prime}$ apart and that ambiguity causes only the restriction of our function to $\Psi_{g}$ to be well-defined.

A diffeomorphism of $S_{g}$ onto a smooth projective curve $C$ determines a natural isomorphism between $\Omega_{g}$ and the space of theta characteristics of $C$ (i.e., square roots of the canonical bundle $K_{C}$; see for instance Appendix B of [2]). This suggests that Johnson's computation should have an algebro-geometric interpretation, if not interesting applications to the geometry of curves.

Problem 7.7. Give an algebro-geometric construction of the epimorphism $T_{g} \rightarrow$ $S_{3} \Omega$.

Van Geemen has suggested such a construction (unpublished).

7.1. Torelli space and period space. The group $T_{g}$ acts freely on $\mathcal{X}_{g}$. The quotient $\mathcal{T}_{g}$ is therefore a complex manifold. It is called Torelli space. According to the discussion at the beginning of Section $3, \mathcal{T}_{g}$ is then a classifying space for $T_{g}$ so that there is a canonical isomorphism $H_{\bullet}\left(T_{g} ; \mathbb{Z}\right) \cong H_{\bullet}\left(\mathcal{T}_{g} ; \mathbb{Z}\right)$. Torelli space has a moduli interpretation; it is the moduli space of smooth projective curves $C$ of genus $g$ together with a symplectic isomorphism

$$
\gamma: H_{1}\left(S_{g} ; \mathbb{Z}\right) \rightarrow H_{1}(C ; \mathbb{Z})
$$

There are also decorated versions $\mathcal{T}_{g, r}^{n}$ of Torelli space. Their points are points of $\mathcal{M}_{g, r}^{n}$ together with a symplectic isomorphism $\gamma$ of $H_{1}(S ; \mathbb{Z})$ with the first homology of the curve corresponding to the point of $\mathcal{M}_{g}$. It is clear that the map $\mathcal{T}_{g, r}^{n} \rightarrow \mathcal{M}_{g, r}^{n}$ is Galois with Galois group $S p_{g}(\mathbb{Z})$.

Denote the Siegel space associated to $V_{g}$ by $\mathfrak{h}_{g}$. To be precise, $\mathfrak{h}_{g}$ is the set the pure Hodge structures on $V_{g}$ with Hodge numbers $(-1,0)$ and $(0,-1)$, polarized by the intersection form. This is a contractible complex manifold of dimension $g(g+1) / 2$ on which the group $S p_{g}(\mathbb{R})$ acts properly and transitively. We can also regard $\mathfrak{h}_{g}$ as the moduli space of pairs consisting of a $g$ dimensional principally polarized abelian variety $A$ plus a symplectic isomorphism

$$
\gamma: H_{1}(S ; \mathbb{Z}) \rightarrow H_{1}(A, \mathbb{Z})
$$

This interprets the $S p_{g}(\mathbb{Z})$ orbit space of $\mathfrak{h}_{g}$ as the moduli space of principally polarized abelian varieties of dimension $g, \mathcal{A}_{g}$. We regard $\mathcal{A}_{g}$ as an orbifold with orbifold fundamental group $S p_{g}(\mathbb{Z})$, although $S p_{g}(\mathbb{Z})$ does not act faithfully on $\mathfrak{h}_{g}$. The kernel of this action is $\{ \pm 1\}$.

Assigning to a smooth projective curve the Hodge structure on its first homology group defines a map $\mathcal{T}_{g} \rightarrow \mathfrak{h}_{g}$, the period map for $T_{g}$. It is an isomorphism in genus 1 , an open imbedding when $g=2$, and $2: 1$ with ramification along the hyperelliptic 
locus when $g \geq 3$.7 The reason for this is that for all abelian varieties we have the equality

$$
[A ; \gamma]=[A ;-\gamma]
$$

of points of $\mathfrak{h}_{g}$ as - id is an automorphism of each abelian variety. On the other hand, we have the equality

$$
[C ; \gamma]=[C ;-\gamma]
$$

of points of $\mathcal{T}_{g}$ if and only if $C$ is hyperelliptic.

Mess's result (mentioned at the beginning of the section) can now be deduced from this: $\mathcal{T}_{2}$ is the complement in $\mathfrak{h}_{2}$ of the locus of principally polarized abelian varieties that are products of two elliptic curves. The locus of such reducible abelian varieties is a countable disjoint union of copies of $\mathfrak{h}_{1} \times \mathfrak{h}_{1}$. The group $S p_{2}(\mathbb{Z})$ permutes them transitively, and each is stabilized by a product of two copies of $S L_{2}(\mathbb{Z})$ and an involution that switches the two copies of the upper half plane. Mess's result follows easily using the stratified Morse theory of Goresky and MacPherson - use distance from a generic point of $\mathfrak{h}_{2}$ as the Morse function. Since each component of $\mathfrak{h}_{2}-\mathcal{T}_{2}$ is a totally geodesic divisor, the distance function has a unique critical point (necessarily a minimum) on each stratum. It follows that $\mathcal{T}_{2}$ has the homotopy type of a wedge of circles, one for each component of $\mathfrak{h}_{2}-\mathcal{T}_{2}$.

The period map gives, after passage to $S p_{g}(\mathbb{Z})$ orbit spaces, a morphism $\mathcal{M}_{g} \rightarrow$ $\mathcal{A}_{g}$, the period mapping for $\mathcal{M}_{g}$. This period mapping extends to the partial completion $\widetilde{\mathcal{M}}_{g} \supset \mathcal{M}_{g}$ and the resulting map $\widetilde{\mathcal{M}}_{g} \rightarrow \mathcal{A}_{g}$ is proper.

Now assume $g \geq 3$ and denote the image of the period map $\mathcal{T}_{g} \rightarrow \mathfrak{h}_{g}$ by $\mathcal{S}_{g}$. This space is the quotient of $\mathcal{T}_{g}$ by the subgroup $\{ \pm 1\} \subset S p_{g}(\mathbb{Z})$. Consequently

$$
H^{\bullet}\left(\mathcal{S}_{g}\right) \cong H^{\bullet}\left(T_{g}\right)^{\{ \pm 1\}} .
$$

Observe that $\mathcal{S}_{g}$ is a locally closed analytic subvariety of $\mathfrak{h}_{g}$, but not closed. The $\{ \pm 1\}$ cover $\mathcal{T}_{g} \rightarrow \mathcal{S}_{g}$ extends as a $\{ \pm 1\}$ cover $\overline{\mathcal{T}}_{g} \rightarrow \overline{\mathcal{S}}_{g}$ over the closure of $\mathcal{S}_{g}$ in $\mathfrak{h}_{g}$ and the $\{ \pm 1\}$ action on the total space is the restriction of an $S p_{g}(\mathbb{Z})$ action. Both $\overline{\mathcal{T}}_{g}$ and $\overline{\mathcal{S}}_{g}$ are rather singular along the added locus (which is of codimension 3 ). If we pass to $S p_{g}(\mathbb{Z})$ orbit spaces, then the natural map

$$
\widetilde{\mathcal{M}}_{g} \rightarrow S p_{g}(\mathbb{Z}) \backslash \overline{\mathcal{T}}_{g} \cong S p_{g}(\mathbb{Z}) \backslash \overline{\mathcal{S}}_{g}
$$

resolves these singularities in an orbifold sense.

It follows from (7.1) that the fundamental group of $\overline{\mathcal{T}}_{g}$ is abelian and is $S p_{g}(\mathbb{Z})$ equivariantly isomorphic to $\wedge^{3} H_{1}\left(S_{g}, \mathbb{Z}\right) /\left(\left[S_{g}\right] \wedge H_{1}\left(S_{g}, \mathbb{Z}\right)\right)$.

Problem 7.8. Understand the topology of $\mathcal{S}_{g}$ and its closure $\overline{\mathcal{S}}_{g}$ in $\mathfrak{h}_{g}$. In particular, how close is $\mathcal{S}_{g}$ to being a finite complex? (Observe that if it has a finite 2-skeleton, then $T_{g}$ is finitely presented.)

Related, but formally independent of this problem, is the question of whether the cohomology of $T_{g}$ stabilizes in a suitable sense:

Question 7.9. Is $H^{k}\left(T_{g}\right)$ expressible as an $S p_{g}(\mathbb{Z})$ module in a manner that is independent of $g$ if $g$ is large enough? For example, from Johnson's Theorem, we know that $H^{1}\left(T_{g}\right)$ is the third fundamental representation of $S p_{g}$ for all $g \geq 3$.

\footnotetext{
${ }^{7}$ It is stated incorrectly in 24 that $\mathcal{T}_{2} \rightarrow \mathfrak{h}_{2}$ is an unramified 2:1 map onto its image.
} 
7.2. The Johnson homomorphism. The proof of Johnson's Theorem is nontrivial and uses geometric topology, but the homomorphism $\tau_{g}^{1}$ is easily described.

Since $T_{g}$ is torsion free, the projection $\mathcal{T}_{g}^{1} \rightarrow \mathcal{T}_{g}$ defines the universal curve over $\mathcal{T}$. Denote the corresponding bundle of jacobians by $\mathcal{J}_{g} \rightarrow \mathcal{T}_{g}$. Since the local system of first homology groups associated to the universal curve is canonically framed, this jacobian bundle $\mathcal{J}_{g} \rightarrow \mathcal{T}_{g}$ is analytically trivial as a bundle of Lie groups: we have a natural trivializing projection $p: \mathcal{J}_{g} \rightarrow \operatorname{Jac} S_{g}$, where $\operatorname{Jac} S_{g}:=$ $H_{1}\left(S_{g} ; \mathbb{R} / \mathbb{Z}\right)$ is the "jacobian" of the reference surface.

The usual Abel-Jacobi map, which assigns to an ordered pair of points $(x, y)$ on a smooth curve $C$ the divisor class of $(x)-(y)$, induces a morphism

$$
\mathcal{T}_{g}^{1} \times \mathcal{T}_{g} \mathcal{T}_{g}^{1} \rightarrow \mathcal{J}_{g}
$$

over $\mathcal{T}_{g}$. This provides a correspondence

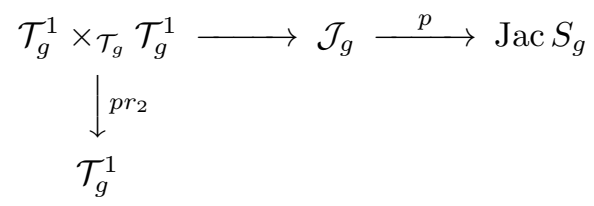

from $\mathcal{T}_{g}^{1}$ to Jac $S_{g}$. It induces homomorphisms

$$
H_{k}\left(T_{g}^{1}\right) \cong H_{k}\left(\mathcal{T}_{g}^{1}\right) \rightarrow H_{k+2}\left(\operatorname{Jac} S_{g}\right)
$$

and the first of these is the Johnson homomorphism

$$
\tau_{g}^{1}: H_{1}\left(T_{g}^{1}\right) \rightarrow H_{3}\left(\operatorname{Jac} S_{g}\right)
$$

for $T_{g}^{1}$. Since $\mathcal{T}_{g}^{1} \rightarrow \mathcal{T}_{g}$ is a fibration of Eilenberg-Maclane spaces, we have an exact sequence of fundamental groups:

$$
1 \rightarrow \pi_{g} \rightarrow T_{g}^{1} \rightarrow T_{g} \rightarrow 1
$$

This induces an exact sequence

$$
H_{1}\left(S_{g} ; \mathbb{Z}\right) \rightarrow H_{1}\left(T_{g}^{1} ; \mathbb{Z}\right) \rightarrow H_{1}\left(T_{g} ; \mathbb{Z}\right) \rightarrow 0
$$

on homology. Since Jac $S_{g}$ is a topological group with torsion free homology, its integral homology has a product - the Pontrjagin product. It is not difficult to check that the composite

$$
H_{1}\left(S_{g} ; \mathbb{Z}\right) \rightarrow H_{1}\left(T_{g}^{1} ; \mathbb{Z}\right) \rightarrow H_{3}\left(\operatorname{Jac} S_{g} ; \mathbb{Z}\right)
$$

is the map given by Pontrjagin product with the class $\left[S_{g}\right]$. It follows that there is a natural homomorphism

$$
H_{1}\left(T_{g} ; \mathbb{Z}\right) \rightarrow H_{3}\left(\operatorname{Jac} S_{g} ; \mathbb{Z}\right) /\left(\left[S_{g}\right] \times H_{1}\left(S_{g} ; \mathbb{Z}\right)\right)
$$

This is the Johnson homomorphism $\tau_{g}$ for $T_{g}$.

Johnson's Theorem, alone and in concert with Saito's theory of Hodge modules, has several interesting applications to the geometry of moduli spaces of curves. 
7.3. Picard groups of level covers. Denote the moduli space of smooth projective genus $g$ curves with a level $l$ structure by $\mathcal{M}_{g}[l]$. This is convenient shorthand for the notation $\mathcal{M}_{g}\left[S p_{g}(\mathbb{Z} / l \mathbb{Z})\right]$ introduced in Section 3. Denote the kernel of the reduction mod $l$ map

$$
S p_{g}(\mathbb{Z}) \rightarrow S p_{g}(\mathbb{Z} / l \mathbb{Z})
$$

by $S p_{g}(\mathbb{Z})[l]$, and its full inverse image in $\Gamma_{g}$ by $\Gamma_{g}[l]$. Then $\mathcal{M}_{g}[l]$ is the quotient of Teichmüller space $\mathcal{X}_{g}$ by $\Gamma_{g}[l]$. As in the case of $\mathcal{M}_{g}$, there is a canonical isomorphism

$$
H^{\bullet}\left(\mathcal{M}_{g}[l]\right) \cong H^{\bullet}\left(\Gamma_{g}[l]\right)
$$

This holds with rational coefficients for all $l$, and arbitrary coefficients whenever $\Gamma_{g}[l]$ is torsion free, which holds whenever $S p_{g}(\mathbb{Z})[l]$ is torsion free $-l \geq 3$.

We know from (7.4) that

$$
H^{1}\left(\Gamma_{g}[l]\right) \cong H^{1}\left(\mathcal{M}_{g}[l]\right)=0
$$

when $g \geq 3$. By standard arguments (cf. [24, §5]), this implies that

$$
c_{1}: \operatorname{Pic} \mathcal{M}_{g}[l] \otimes \mathbb{Q} \rightarrow H^{2}\left(\mathcal{M}_{g}[l]\right)
$$

is injective, and therefore that $\operatorname{Pic} \mathcal{M}_{g}[l]$ is finitely generated when $g \geq 3$.

The stable cohomology of an arithmetic group depends only on the ambient real algebraic group [5]. Based on this, one might expect that the natural map

$$
H^{k}\left(\Gamma_{g}\right) \rightarrow H^{k}\left(\Gamma_{g}[l]\right)
$$

is an isomorphism for all $l \geq 0$, once the genus $g$ is sufficiently large compared to the degree $k$. It follows from Johnson's work that this is true when $k=1$ (cf. $24)$, but the only evidence for it when $k>1$ is Harer's computation of the second homology of the spin mapping class groups [33], and Foisy's theorem from which Harer's computation now follows:

Theorem 7.10 (Foisy [15]). For all $g \geq 3$, the natural map $H^{2}\left(\Gamma_{g}\right) \rightarrow H^{2}\left(\Gamma_{g}[2]\right)$ is an isomorphism. Consequently, Pic $\mathcal{M}_{g}[2]$ is finitely generated of rank 1.

Question 7.11. Is Pic $\mathcal{M}_{g}[l]$ rank 1 for all $g \geq 3$ and all $l \geq 1$ ?

This would be the case if we knew that the $S p_{g}(\mathbb{Z})$ action on $H^{2}\left(T_{g}\right)$ extended to an algebraic action of $S p_{g}$, for we could then invoke Borel's computation of the stable cohomology of arithmetic groups [5].

7.4. Normal Functions. Each rational representation $V$ of $S p_{g}$ gives rise to an orbifold local system $\mathbb{V}$ over $\mathcal{M}_{g}[l]$. Such a a local system underlies an admissible variation of Hodge structure. First, if $V$ is irreducible, then $V$ underlies a variation of Hodge structure unique up to Tate twist ([24, (9.1)]). Every polarized $\mathbb{Q}$ variation of Hodge structure whose monodromy representation comes from a rational representation of $S p_{g}$ has the property that each of its isotypical components is an admissible variation of Hodge structure of the form $A_{\lambda} \otimes \mathbb{V}(\lambda)$, where $A_{\lambda}$ is a Hodge structure and $\mathbb{V}(\lambda)$ is a variation of Hodge structure corresponding to the $S p_{g}$ module with highest weight $\lambda$ - cf. [24, (9.2)].

For a Hodge structure $V$ of weight -1 one defines the corresponding intermediate jacobian $J V$ by

$$
J V=V_{\mathbb{C}} /\left(F^{0} V+V_{\mathbb{Z}}\right) .
$$


Its interest comes from the fact that it parametrizes the extensions of $\mathbb{Z}$ by $V$ in the MHS category: if $E$ is an extension of $\mathbb{Z}$ by $V$, then choose an integral lift $e \in E$ of 1 and consider the image of $e$ in

$$
E_{\mathbb{C}} /\left(F^{0} E+V_{\mathbb{Z}}\right) \cong V_{\mathbb{C}} /\left(F^{0} V+V_{\mathbb{Z}}\right) .
$$

This is independent of the lift and yields a complete invariant of the extension. There is converse construction that makes $J V$ support a variation of mixed Hodge structure $\mathbb{E}$ that is universal as an extension of the trivial Hodge structure $\mathbb{Z}$ by the constant Hodge structure $V$ :

$$
0 \rightarrow \mathbb{V}_{J V} \rightarrow \mathbb{E} \rightarrow \mathbb{Z}_{J V} \rightarrow 0
$$

(see $[7$ ). This immediately generalizes to a relative setting: if $\mathbb{V}$ is an admissible variation of $\mathbb{Z}$ Hodge Structure of weight -1 over a smooth variety $X$, then we have a corresponding bundle $\pi: \mathcal{J V} \rightarrow X$ of intermediate jacobians over $X$ supporting a universal extension

$$
0 \rightarrow \pi^{*} \mathbb{V} \rightarrow \mathbb{E} \rightarrow \pi^{*} \mathbb{Z}_{X} \rightarrow 0 .
$$

A section $\sigma$ of $\mathcal{J} \mathbb{V}$ over $X$ determines an extension of Hodge structures:

$$
0 \rightarrow \mathbb{V} \rightarrow \sigma^{*} \mathbb{E} \rightarrow \mathbb{Z}_{X} \rightarrow 0
$$

A normal function is a section of $\mathcal{J} \mathcal{V}$ such that the corresponding extension $\mathbb{E}$ is an admissible variation of mixed Hodge structure. The normal functions arising from algebraic cycles are normal functions in this sense - cf. [24, §6].

We briefly recall Griffiths' construction of a normal function associated to a family of homologically trivial algebraic cycles. First we consider the case where the base is a point. Suppose that $X$ is a smooth projective variety. A homologically trivial algebraic $d$-cycle $Z$ in $X$ canonically determines an extension of $\mathbb{Z}$ by $H_{2 d+1}(X, \mathbb{Z}(-d))$ by pulling back the exact sequence

$$
0 \rightarrow H_{2 d+1}(X ; \mathbb{Z}(-d)) \rightarrow H_{2 d+1}(X,|Z| ; \mathbb{Z}(-d)) \rightarrow H_{2 d}(|Z| ; \mathbb{Z}(-d)) \rightarrow \cdots
$$

of MHSs along the inclusion

$$
\mathbb{Z} \rightarrow H_{2 d}(|Z|, \mathbb{Z}(-d))
$$

that takes 1 to the class of $Z$. So an integral lift of 1 is given by an integral singular $2 d+1$ chain $\Gamma$ in $X$ whose boundary is $Z$. Integration identifies $J H_{2 d+1}(X ; \mathbb{Z}(-d))$ with the Griffiths intermediate Jacobian

$$
J_{d}(X):=\operatorname{Hom}_{\mathbb{C}}\left(F^{d} H^{2 d+1}(X) ; \mathbb{C}(-d)\right) / H^{2 d+1}(X ; \mathbb{Z}(-d)),
$$

and under this isomorphism the extension class in question is just given by integration over $\Gamma$.

Families of homologically trivial cycles give rise to normal functions: Suppose that $\mathcal{X} \rightarrow T$ is a family of smooth projective varieties over a smooth base $T$ and that $\mathcal{Z}$ is an algebraic cycle in $\mathcal{X}$ which is proper over $T$ of relative dimension $d$. Then the local system whose fiber over $t \in T$ is $H_{2 d+1}\left(X_{t}, \mathbb{Z}(-d)\right)$ naturally underlies a variation of Hodge structure $\mathbb{V}$ over $T$ of weight -1 so that we can form the $d$ th relative intermediate jacobian

$$
\mathcal{J}_{d}(\mathcal{X} / T) \rightarrow T
$$

whose fiber over $t \in T$ is $J_{d}\left(X_{t}\right)$. The family of cycles $\mathcal{Z}$ defines a section this bundle which is a normal function. 
Theorem 7.12 (Hain 24]). Suppose that $\mathbb{V}$ is an admissible variation of Hodge structure of weight -1 over $\mathcal{M}_{g}[l]$ whose monodromy representation factors through a rational representation of $S p_{g}$. If $g \geq 3$, then the space of normal functions associated to $\mathbb{V}$ is finitely generated of rank equal to the number of copies of the variation $\mathbb{V}\left(\lambda_{3}\right)$ of weight -1 that occur in $\mathbb{V}$.

The theorem implies that, up to torsion and multiples, there is only one normal function over $\mathcal{M}_{g}$ associated to a variation of Hodge structure whose monodromy factors through a rational representation of $S p_{g}$. So what is the generator of these normal functions?

To answer this question, recall that if $C$ is a smooth projective curve of genus $g$ and $x \in C$, we have the Abel-Jacobi morphism

$$
C \rightarrow \operatorname{Jac} C, \quad y \mapsto(y)-(x) .
$$

Denote the image 1-cycle in $\operatorname{Jac} C$ by $C_{x}$ and the cycle $i_{*} C_{x}$ by $C_{x}^{-}$, where $i$ : Jac $C \rightarrow \operatorname{Jac} C$ takes $u$ to $-u$. The cycle $C_{x}-C_{x}^{-}$is homologous to zero, and therefore defines a point $\nu^{1}(C, x)$ in $J_{1}(\operatorname{Jac} C)$. Pontrjagin product with the class of $C$ induces a homomorphism

$$
A: \operatorname{Jac} C \rightarrow J_{1}(\mathrm{Jac} C) .
$$

We call the cokernel of $A$ the primitive first intermediate Jacobian $J_{1}^{\mathrm{pr}}(\mathrm{Jac} C)$ of Jac $C$. The family of such primitive intermediate jacobians over $\mathcal{M}_{g}$ is the unique one (up to isogeny) associated to the variation of Hodge structure of weight -1 over $\mathcal{M}_{g}$ whose associated $\Gamma_{g}$ module is $V\left(\lambda_{3}\right)$. It is not difficult to show that

$$
\nu^{1}(C, x)-\nu^{1}(C, y)=2 A(x-y) .
$$

It follows that the image of $\nu^{1}(C, x)$ in $J_{1}^{\mathrm{pr}}(\operatorname{Jac} C)$ is independent of $x$. The image will be denoted by $\nu(C)$. This is the value of the normal function associated with $C-C^{-}$over $[C]$.

7.5. Picard group of the generic curve with a level $l$ structure. The classification of normal functions allows one to determine the Picard group of the generic point of $\mathcal{M}_{g}[l]$. The case $l=1$ was the subject of the Franchetta Conjecture which was deduced from Harer's computation of $\Gamma_{g}$ by Beauville (unpublished) and by Arbarello and Cornalba [3].

Theorem 7.13 (Hain 25]). The Picard group of the generic curve of genus $g \geq 3$ with a level $l$ structure is of rank 1, has torsion subgroup isomorphic to the l torsion points $H_{1}\left(\mathrm{Jac} S_{g}, \mathbb{Z} / l \mathbb{Z}\right)$, and, modulo torsion, is generated by the canonical bundle if $l$ is odd, and a theta characteristic if $l$ is even.

\section{Relative Malcev Completion}

Fundamental groups of smooth algebraic varieties are quite special as we know from the work of Morgan 62] and others. The least trivial restrictions on these groups come from Hodge theory and Galois theory. Since $\Gamma_{g}$ is the (orbifold) fundamental group of $\mathcal{M}_{g}$, a smooth orbifold, Hodge theory and Galois theory should have something interesting to say about its structure. To put a MHS on a group one needs to linearize it. One way to do this is to replace the group by some kind of algebraic envelope and put a MHS on the coordinate ring of this (pro)algebraic group. 
In this section we introduce these linearizations and use them to establish a relation between the basic normal function and a remarkable central extension that is hidden in a quotient of the mapping class group. Here the impact of mixed Hodge theory is not yet felt, but we are setting the stage for Section 9 where it is omnipresent.

8.1. Classical Malcev completion. Suppose that $\pi$ is a finitely generated group. The classical Malcev (or unipotent) completion of $\pi$ consists of a prounipotent group $\mathcal{U}(\pi)$ (over $\mathbb{Q}$ ) and a homomorphism $\pi \rightarrow \mathcal{U}(\pi)$. It is characterized by the following universal mapping property: if $U$ is a unipotent group, and $\phi: \pi \rightarrow U$ is a homomorphism, there is a unique homomorphism of prounipotent groups $\mathcal{U}(\pi) \rightarrow U$ through which $\phi$ factors. There are several well known constructions of the unipotent completion, which can be found in [23], for example. Each (pro)unipotent group $U$ is isomorphic to its Lie algebra $\mathfrak{u}$, a (pro)nilpotent Lie algebra via the exponential map. Thus, to give the Malcev group $\mathcal{U}(\pi)$ associated to $\pi$ it suffices to give its associated pronilpotent Lie algebra $\mathfrak{u}(\pi)$. This Lie algebra is called the Malcev Lie algebra associated to $\pi$. It comes with a natural descending filtration whose $k$ th term $\mathfrak{u}^{(k)}(\pi)$ is the closed ideal of $\mathfrak{u}(\pi)$ of generated by its $k$-fold commutators $(k=1,2, \ldots)$ and it is complete with respect to this filtration. We will refer to this filtration as the Malcev filtration.

When $\pi$ is the fundamental group $\pi_{1}(X, x)$ of a smooth complex algebraic variety, $\mathfrak{u}(\pi)$ has a canonical MHS which was first constructed by Morgan 62. If $X$ is also complete, or more generally, when $H_{1}(X)$ has a pure Hodge structure of weight -1 , then the weight filtration is the Malcev filtration:

$$
W_{-k} \mathfrak{u}\left(\pi_{1}(X, x)\right)=\mathfrak{u}^{(k)}\left(\pi_{1}(X, x)\right) .
$$

Alternatively, this MHS determines and is determined by a MHS on the coordinate ring $\mathcal{O}(\mathcal{U}(\pi))$ of the associated Malcev group.

We shall denote the Malcev completion of $\pi_{g}^{n}=\pi_{1}\left(S_{g}^{n}, x_{0}\right)$ by $\mathfrak{p}_{g}^{n}$.

8.2. Relative Malcev completion. The Malcev completion of a group $\pi$ is trivial when $H_{1}(\pi)$ vanishes, for then $\pi$ has no non-trivial unipotent quotients. Since the first homology of $\Gamma_{g}$ vanishes for all $g$, its Malcev completion will be trivial. Deligne has defined the notion of Malcev completion of a group $\pi$ relative to a Zariski dense homomorphism $\rho: \pi \rightarrow S$, where $S$ is a reductive algebraic group defined over a base field $F$ (that we assume to be of characteristic zero).

The Malcev completion of $\pi$ relative to $\rho: \pi \rightarrow S$ is a a proalgebraic $F$-group $\mathcal{G}(\pi, \rho)$, which is an extension

$$
1 \rightarrow \mathcal{U} \rightarrow \mathcal{G}(\pi, \rho) \rightarrow S \rightarrow 1
$$

of $S$ by a prounipotent group, together with a lift $\tilde{\rho}: \pi \rightarrow \mathcal{G}(\pi, \rho)$ of $\rho$. \$It is characterized by the following universal mapping property: if $G$ is an $F$-group which is an extension of $S$ by a unipotent group $U$, and if $\phi: \pi \rightarrow G$ is a homomorphism, then there is a unique homomorphism $\mathcal{G}(\pi, \rho) \rightarrow G$ through which $\phi$ factors:

$$
\phi: \pi \stackrel{\tilde{\rho}}{\rightarrow} \mathcal{G}(\pi, \rho) \rightarrow G
$$

\footnotetext{
${ }^{8}$ In many cases the completion of $\pi$ over an algebraic closure $\bar{F}$ of $F$ is the set of $\bar{F}$ points of the completion of $\pi$ over $F$. This is the case for the mapping class groups when $g \geq 3$, but we do not know this is true in general, except when $S$ is trivial.
} 
Since $S$ is reductive, we should think of $\mathcal{U}$ as the prounipotent radical of $\mathcal{G}(\pi, \rho)$. One can show for instance that $\mathcal{U}$ has a Levi supplement so that $\mathcal{G}(\pi, \rho)$ is a semidirect product of $S$ and $\mathcal{U}$. The Lie algebra $\mathfrak{g}(\pi, \rho)$ of $\mathcal{G}(\pi, \rho)$ also comes with a Malcev filtration with respect to which it is complete: $\mathfrak{g}(\pi, \rho)^{(0)}=\mathfrak{g}(\pi, \rho)$, and for $k \geq 1, \mathfrak{g}(\pi, \rho)^{(k)}$ is the closed ideal generated by $k$-fold commutators in the Lie algebra of $\mathcal{U}$.

We will often write $\mathcal{G}(\pi)$ instead of $\mathcal{G}(\pi, \rho)$ when the representation $\rho$ is clear from the context. We shall denote the completion of the (orbifold) fundamental group of a pointed orbifold $(X, x)$ with respect to a Zariski dense reductive representation $\rho: \pi_{1}(X, x) \rightarrow S$ by $\mathcal{G}(X, x ; \rho)$, or simply $\mathcal{G}(X, x)$ when $\rho$ is clear from the context.

When $S$ is trivial, we recover the classical Malcev completion. The universal property of the Malcev completion of ker $\rho$ yields a natural homomorphism of proalgebraic $F$-groups $\mathcal{U}(\operatorname{ker} \rho)(F) \rightarrow \mathcal{G}(\pi)$. In general, it is neither surjective nor injective in general as the following two examples show.

Example 8.1. The fundamental group of the symplectic Lie group $S p_{g}(\mathbb{R})$ is infinite cyclic and hence so is its universal cover $\widehat{S p}_{g}(\mathbb{R}) \rightarrow S p_{g}(\mathbb{R})$. This universal cover is not an algebraic group (which follows for instance from the fact that the complexification of $S p_{g}(\mathbb{R}), S p_{g}(\mathbb{C})$, is simply connected). The preimage $\widehat{S p}_{g}(\mathbb{Z})$ of $S p_{g}(\mathbb{Z})$ in this covering contains the universal central extension of $S p_{g}(\mathbb{Z})$ by $\mathbb{Z}$. Now take for $\pi$ this central extension and for $\rho$ its natural homomorphism to $S p_{g}(\mathbb{C})$. The corresponding relative Malcev completion is then reduced to $S p_{g}(\mathbb{C})$ itself, so that the homomorphism from $\mathcal{U}(\mathbb{Z})(\mathbb{C})$ (which is just the abelian group $\mathbb{C}$ ) to $\mathcal{G}\left(\widehat{S p}_{g}(\mathbb{Z})\right)$ is trivial. We will see that this example is realized inside a quotient of the mapping class group.

Example 8.2. In this example, $\operatorname{ker} \rho$ is trivial, but $\mathcal{U}(\pi)$ is not. The basic fact we need (see [25]) is that there is always a natural $S$ equivariant isomorphism

$$
H_{1}(\mathfrak{u}(\pi)) \cong \oplus_{\alpha \in \check{S}} H_{1}\left(\pi, V_{\alpha}\right) \otimes V_{\alpha}^{*},
$$

where $V_{\alpha}$ denotes a representation with highest weight $\alpha$. For $\pi$ we take $\Gamma$, a finite index subgroup of $S L_{2}(\mathbb{Z})$, for $S$ we take $S L_{2}(\mathbb{Q})$, and for $\rho$ we take the natural inclusion. Denote the $n$th power of the fundamental representation of $S L_{2}$ by $S^{n} V$. For all such $\Gamma$, there is an infinite number of integers $n \geq 0$ such that $H^{1}\left(\Gamma, S^{n} V\right)$ is non-trivial. 9 It follows that $\mathcal{U}(\Gamma)$ has an infinite dimensional $H_{1}$, even though $\operatorname{ker} \rho$ is trivial.

This example suggests the following problem:

Problem 8.3. Investigate the relationship between the theory of modular forms associated to a finite index subgroup $\Gamma$ of $S L_{2}(\mathbb{Z})$ and the completion of $\Gamma$ relative to the inclusion $\Gamma \hookrightarrow S L_{2}(\mathbb{Q})$.

8.3. The relative Malcev completion of $\Gamma_{g}$. The natural homomorphism $\rho$ : $\Gamma_{g, r}^{n} \rightarrow S p_{g}$ has Zariski dense image. Denote the completion of $\Gamma_{g, r}^{n}$ relative to $\rho$ by $\mathcal{G}_{g, r}^{n}$, its prounipotent radical by $\mathcal{U}_{g, r}^{n}$ and their Lie algebras by $\mathfrak{g}_{g, r}^{n}$ and $\mathfrak{u}_{g, r}^{n}$.

The following theorem indicates the presence of exactly one copy of the universal central extension of $S p_{g}(\mathbb{Z})$ in a mapping class group of genus $g$ when $g \geq 3$.

\footnotetext{
${ }^{9}$ This is easily seen when $\Gamma$ is free, for example. In general it is related to the theory of modular forms.
} 
Theorem 8.4 (Hain [23]). When $g \geq 2$, the homomorphism

$$
\mathcal{U}\left(T_{g, r}^{n}\right) \rightarrow \mathcal{U}_{g, r}^{n}
$$

is surjective. When $g \geq 3$, its kernel is a central subgroup isomorphic to the additive group.

This phenomenon is intimately related to the cycle $C-C^{-}$and its normal function as we shall now explain.

8.4. The central extension. The existence of the central extension has both a group theoretic and a geometric explanation. It is also related to the Casson invariant through the work of Morita [65, 66]. We begin with the group theoretic one.

The group analogue of the Malcev filtration for the Torelli group $T_{g}$ is the most rapidly descending central series of $T_{g}$ with torsion free quotients:

$$
T_{g}=T_{g}^{(1)} \supset T_{g}^{(2)} \supset T_{g}^{(3)} \supset \cdots
$$

Note that $T_{g}^{(1)} / T_{g}^{(2)}$ is the maximal torsion free abelian quotient of $T_{g}$, which is

$$
V\left(\lambda_{3}\right)_{\mathbb{Z}}:=\wedge^{3} H_{1}\left(S_{g} ; \mathbb{Z}\right) /\left(\left[S_{g}\right] \times H_{1}\left(S_{g} ; \mathbb{Z}\right)\right)
$$

by Johnson's Theorem (7.1). The group $\Gamma_{g} / T_{g}^{(3)}$ can be written as an extension

$$
1 \rightarrow T_{g}^{(2)} / T_{g}^{(3)} \rightarrow \Gamma_{g} / T_{g}^{(3)} \rightarrow \Gamma_{g} / T_{g}^{(2)} \rightarrow 1 .
$$

It turns out that this sequence contains a multiple of the universal central extension of $S p_{g}(\mathbb{Z})$ by $\mathbb{Z}$.

Since $V\left(\lambda_{3}\right)$ is a rational representation of $S p_{g}$, and since the surjection

$$
\wedge^{2} V\left(\lambda_{3}\right)_{\mathbb{Z}} \rightarrow T_{g}^{(2)} / T_{g}^{(3)}
$$

induced by the commutator is $S p_{g}(\mathbb{Z})$ equivariant, it follows that $T_{g}^{(2)} / T_{g}^{(3)} \otimes \mathbb{Q}$ is also a rational representation of $S p_{g}$. Because $V\left(\lambda_{3}\right)$ is an irreducible symplectic representation, there is exactly one copy of the trivial representation in $\wedge^{2} V\left(\lambda_{3}\right)$. This copy of the trivial representation survives in $T_{g}^{(2)} / T_{g}^{(3)} \otimes \mathbb{Q} 23$ so that there is an $S p_{g}(\mathbb{Z})$ equivariant projection $T_{g}^{(2)} / T_{g}^{(3)} \rightarrow \mathbb{Z}$. Pushing the extension (8.2) out along this map gives an extension

$$
0 \rightarrow \mathbb{Z} \rightarrow E \rightarrow \Gamma_{g} / T_{g}^{(2)} \rightarrow 1
$$

Note that $E$ is a quotient of $\Gamma_{g}$. We will manufacture a multiple of the universal central extension of $S p_{g}(\mathbb{Z})$ from this group that turns out to be the obstruction to the map $\mathcal{U}\left(T_{g}\right) \rightarrow \mathcal{U}_{g}$ being injective. (Full details can be found in [23].)

The group $\Gamma_{g} / T_{g}^{(2)}$ can be written as an extension

$$
0 \rightarrow V\left(\lambda_{3}\right)_{\mathbb{Z}} \rightarrow \Gamma_{g} / T_{g}^{(2)} \rightarrow S p_{g}(\mathbb{Z}) \rightarrow 1 .
$$

Morita [67] showed that this extension is semisplit, that is, if we replace $V\left(\lambda_{3}\right)_{\mathbb{Z}}$ by $\frac{1}{2} V\left(\lambda_{3}\right)_{\mathbb{Z}}$, it splits. (As we shall see, this can also be seen using the normal function of $C-C^{-}$.)

Theorem 8.5 (Morita [66], Hain [23]). The extension of $S p_{g}(\mathbb{Z})$ by $\mathbb{Z}$ obtained by pulling back the extension (8.3) along a semisplitting of (8.4) contains the universal central extension of $S p_{g}(\mathbb{Z})$. 
The geometric picture uses the normal function of $C-C^{-}$. The period map $\mathcal{M}_{g} \rightarrow \mathcal{A}_{g}$ induces the homomorphism $\Gamma_{g} \rightarrow S p_{g}(\mathbb{Z})$. Over $\mathcal{A}_{g}$, we have the orbifold local system $\mathbb{V}\left(\lambda_{3}\right)_{\mathbb{Z}}$ whose fiber over the point corresponding to the abelian variety $A$ polarized by $\theta \in H_{2}(A ; \mathbb{Z}(-1))$ is $H_{3}(A ; \mathbb{Z}(-1)) / \theta \times H_{1}(A ; \mathbb{Z})$. This is a variation of Hodge structure of weight -1 . There is a corresponding bundle of intermediate jacobians

$$
\mathcal{J} \mathbb{V}\left(\lambda_{3}\right)_{\mathbb{Z}} \rightarrow \mathcal{A}_{g}
$$

The normal function $C \mapsto \nu(C)$ gives a lift $\mathcal{M}_{g} \rightarrow \mathcal{J} \mathbb{V}\left(\lambda_{3}\right)_{\mathbb{Z}}$ of the period map, which we also denote by $\nu$. It is easily seen to extend over the partial completion $\widetilde{\mathcal{M}}_{g}$ :

$$
\tilde{\nu}: \widetilde{\mathcal{M}}_{g} \rightarrow \mathcal{J} \mathbb{V}\left(\lambda_{3}\right)_{\mathbb{Z}}
$$

The orbifold fundamental group of the bundle of intermediate jacobians is an extension of $S p_{g}(\mathbb{Z})$ by $V\left(\lambda_{3}\right)_{\mathbb{Z}}$ as both the base and fiber are Eilenberg MacLane spaces with these groups as orbifold fundamental groups. The extension is split by the zero section, and so is naturally a semidirect product. But by 7.2 this is also the orbifold fundamental group of $\widetilde{\mathcal{M}}_{g}$. However, the map induced by $\nu$ on fundamental groups is not an isomorphism:

Proposition 8.6. The extended normal function $\tilde{\nu}: \widetilde{\mathcal{M}}_{g} \rightarrow \mathcal{J} \mathbb{V}\left(\lambda_{3}\right)_{\mathbb{Z}}$ induces an injection on orbifold fundamental groups and has image $S p_{g}(\mathbb{Z}) \ltimes 2 V\left(\lambda_{3}\right)_{\mathbb{Z}}$.

So $\nu$ induces twice the Johnson homomorphism $\tau_{g}$. This explains why the extension (8.4) splits after dividing by 2 .

There is a canonical $\mathbb{C}^{\times}$bundle $\mathcal{B}^{\times}$over $\mathcal{J} \mathbb{V}\left(\lambda_{3}\right)_{\mathbb{Z}}$ such that a twist of $\nu^{*} \mathcal{B}$ by a power of the standard line bundle on $\mathcal{M}_{g}$ is the geometric realization of the extension (8.3). This involves a classical construction: Let $\langle$,$\rangle denote the$ integral symplectic form on $V\left(\lambda_{3}\right)_{\mathbb{Z}}$ that comes from the polarization. For any $(v, n) \in V\left(\lambda_{3}\right)_{\mathbb{Z}} \times \mathbb{Z}$ we have an affine transformation in $V\left(\lambda_{3}\right)_{\mathbb{R}} \times \mathbb{C}$ defined by the rule $(z, t) \mapsto(z+v,\langle z, v\rangle+t+n)$. These transformations make up a group $\widehat{V}\left(\lambda_{3}\right)_{\mathbb{Z}}$, so that $V\left(\lambda_{3}\right)_{\mathbb{Z}} \times \mathbb{Z}$ acquires a group structure. This group is a central extension of $V\left(\lambda_{3}\right)_{\mathbb{Z}}$ by $\mathbb{Z}$ and is often referred to as the Heisenberg group of the symplectic lattice $V\left(\lambda_{3}\right)_{\mathbb{Z}}$. Its action on $V\left(\lambda_{3}\right)_{\mathbb{R}} \times \mathbb{C}$ is free and the orbit space $B$ has the structure of a $\mathbb{C}^{\times}$bundle over the compact torus $V\left(\lambda_{3}\right)_{\mathbb{Z}} \otimes \mathbb{R} / \mathbb{Z}$. (The Chern class of this bundle is represented by the image of the symplectic form in $\wedge^{2} \operatorname{Hom}\left(V\left(\lambda_{3}\right)_{\mathbb{Z}}, \mathbb{Z}\right)$, and hence two-divisible; if we want the square root of this bundle, then we must first choose a $\mathbb{F}_{2}$ valued quadratic function relative the symplectic form mod two.)

If $V\left(\lambda_{3}\right)_{\mathbb{R}}$ comes with a complex structure $J$ that leaves the symplectic form invariant, then we modify the action in an innocent (and standard) way to make it a complex action:

$$
(v, n) \cdot(z, t):=(z+v,\langle z, v\rangle-\sqrt{-1}\langle J z+2 J v, v\rangle+t+n) .
$$

The resulting $\mathbb{C}^{\times}$bundle over the complex torus $J V\left(\lambda_{3}\right)_{\mathbb{Z}}$ is then holomorphic.

Notice that each element of $\mathfrak{h}_{g}$ determines such a complex structure $J$ as above. The naturality of this construction guarantees that it is $S p_{g}(\mathbb{Z})$ equivariant. So if we let $S p_{g}(\mathbb{Z})$ act on $\mathfrak{h}_{g} \times V\left(\lambda_{3}\right)_{\mathbb{R}} \times \mathbb{C}$ as usual on the first two factors and trivially on the second, then there results a natural action of the semidirect product $S p_{g}(\mathbb{Z}) \ltimes \widehat{V}\left(\lambda_{3}\right)_{\mathbb{Z}}$ on this space whose orbit space is in a natural way an orbifold 
$\mathbb{C}^{\times}$bundle over $\mathcal{J} \mathbb{V}\left(\lambda_{3}\right)_{\mathbb{Z}}$. This is our $\mathcal{B}^{\times}$. Its orbifold fundamental group is $S p_{g}(\mathbb{Z}) \ltimes \widehat{V}\left(\lambda_{3}\right) \cdot 10$

Notice that the $\mathbb{C}^{\times}$bundle $\tilde{\nu}^{*} \mathcal{B}^{\times}$has orbifold fundamental group which is a central extension of $\Gamma_{g} / T_{g}^{(2)}$. This central extension is nontrivial, even when restricted to $\mathcal{M}_{g}$ :

Proposition 8.7 (Hain [23]). The central extension of $\Gamma_{g} / T_{g}^{(2)}$ defined by the orbifold fundamental group of the $\mathbb{C}^{\times}$bundle $\tilde{\nu}^{*} \mathcal{B}^{\times}$maps onto a finite index subroup of the central extension (8.3). In particular, both $\tilde{\nu}^{*} \mathcal{B}^{\times}$and $\nu^{*} \mathcal{B}^{\times}$have nonzero rational first Chern class (and so $\nu$ does not lift to a map $\mathcal{M}_{g} \rightarrow \mathcal{B}^{\times}$).

The bundle $\nu^{*} \mathcal{B}^{\times}$is canonically metrized and, as such, is the $\mathbb{C}^{\times}$bundle associated to the archimedean height of the cycles $C-C^{-}$, cf. [23].

\section{Hodge Theory of the Mapping Class Group}

One reason that mixed Hodge theory is so powerful is that the MHS category is abelian. In many situations this turns out to have topological implications for algebraic varieties that are difficult, if not impossible, to obtain directly. A somewhat related (but less exploited) property is that a MHS is canonically split over $\mathbb{C}$. This implies that the weight filtration (which often has a topological interpretation) splits in a way that is compatible with all the algebraic structure naturally present. So, for many purposes, there is no loss of information regarding this algebraic structure if we pass to the corresponding weight graded object. For example, the Malcev filtration on the Malcev Lie algebra of a smooth projective variety is minus the weight filtration, and it therefore splits over $\mathbb{C}$ in a natural way. This splitting is natural in the sense that it respects the Lie algebra structure and is preserved under all base point preserving morphisms. But if we vary the complex structure on $X$ or the base point $x$, then the splitting will, in general, vary with it.

A basic example is the Malcev Lie algebra $\mathfrak{p}_{g}^{1}$ of $\pi_{g}^{1}=\pi_{1}\left(S_{g}^{1}, x_{0}\right)$. The group $\pi_{g}^{1}$ is free on $2 g$ generators and it is classical fact (and easy to show) that the graded of $\mathfrak{p}_{g}^{1}$ with respect to the Malcev filtration is just the free Lie algebra generated by $V_{g}$. If $S_{g}$ is given a conformal structure, then $V_{g}$ has a pure Hodge structure of weight -1 and the weight filtration of $\mathfrak{p}_{g}^{1}$ is minus the Malcev filtration. The splitting allows us now to identify $\mathfrak{p}_{g}^{1} \otimes \mathbb{C}$ with the completion of $\operatorname{Lie}\left(V_{g}\right) \otimes \mathbb{C}$.

We shall come back to this example in Section 10.3. But for now we will focus on the relative Malcev completions introduced in the previous section.

9.1. Hodge theory of $\mathcal{G}_{g, r}^{n}$. A choice of a conformal structure on $S_{g}$ and nonzero tangent vectors at $x_{n+1}, \ldots, x_{n+r}$, determines a point $x_{o}$ of moduli space $\mathcal{M}_{g, r}^{n}$. We can thus identify $\Gamma_{g, r}^{n}$ with the orbifold fundamental group of $\left(\mathcal{M}_{g, r}^{n}, x_{o}\right)$. This induces an isomorphism of $\mathcal{G}_{g, r}^{n}$ with $\mathcal{G}\left(\mathcal{M}_{g, r}^{n}, x_{o}\right)$, the completion of $\pi_{1}\left(\mathcal{M}_{g, r}^{n}, x_{o}\right)$ with respect to the standard symplectic representation. We shall denote $\mathcal{G}\left(\mathcal{M}_{g, r}^{n}, x_{o}\right)$ by $\mathcal{G}_{g, r}^{n}\left(x_{o}\right)$, and its prounipotent radical by $\mathcal{U}_{a r r}^{n}\left(x_{o}\right)$. There is a general Hodge de Rham theory of relative Malcev completion 25. Applying it to $\left(\mathcal{M}_{g, r}^{n}, x_{o}\right)$, one obtains the following result:

\footnotetext{
${ }^{10}$ There is an alternative construction of $\mathcal{B}^{\times}$as a moduli space of biextensions (in the MHS category) with weight graded quotients $\mathbb{Z}, \wedge^{3} H_{1}(A)(-1) / H_{1}(A)$ and $\mathbb{Z}(1)$. Details may be found in 23.
} 
Theorem 9.1 (Hain [25). For each choice of a base point $x_{o}$ of $\mathcal{M}_{g, r}^{n}$, there is a canonical $M H S$ on the coordinate ring $\mathcal{O}\left(\mathcal{G}_{g, r}^{n}\left(x_{o}\right)\right)$ which is compatible with its Hopf algebra structure. Consequently, the Lie algebra $\mathfrak{g}_{g, r}^{n}\left(x_{o}\right)$ of $\mathcal{G}_{g, r}^{n}\left(x_{o}\right)$ and the Lie algebra of its unipotent radical $\mathfrak{u}_{g, r}^{n}\left(x_{o}\right)$ both have a natural MHS.

Denote the Malcev Lie algebra of the subgroup of $\pi_{1}\left(\mathcal{M}_{g, r}^{n}, x_{o}\right)$ corresponding to the Torelli group $T_{g, r}^{n}$ by $\mathfrak{t}_{g, r}^{n}\left(x_{o}\right)$. The normal function of $C-C^{-}$can be used to lift the MHS from $\mathfrak{u}_{g, r}^{n}\left(x_{o}\right)$ to $\mathfrak{t}_{g, r}^{n}\left(x_{o}\right)$.

Theorem 9.2 (Hain [26]). For each $g \geq 3$ and for each choice of a base point $x_{o}$ of $\mathcal{M}_{g, r}^{n}$, there is a canonical MHS on $\mathfrak{t}_{g, r}^{n}\left(x_{o}\right)$ which is compatible with its bracket. Moreover, the canonical central extension

$$
0 \rightarrow \mathbb{Q}(1) \rightarrow \mathfrak{t}_{g, r}^{n}\left(x_{o}\right) \rightarrow \mathfrak{u}_{g, r}^{n}\left(x_{o}\right) \rightarrow 0
$$

is an extension of MHSs, and the weight filtration equals the Malcev filtration.

9.2. A presentation of $\mathfrak{t}_{g}$. We denote the Malcev Lie algebra of $T_{g, r}^{n}$ by $\mathfrak{t}_{g, r}^{n}$. The existence of a MHS on $\mathfrak{t}_{g, r}^{n}\left(x_{o}\right)$ implies that, after tensoring with $\mathbb{C}$, there is a canonical isomorphism

$$
\mathfrak{t}_{g, r}^{n}\left(x_{o}\right) \otimes \mathbb{C} \cong \prod_{m} \operatorname{Gr}_{-m}^{W} \mathfrak{t}_{g, r}^{n}\left(x_{o}\right) \otimes \mathbb{C} .
$$

Since the left hand side is (noncanonically) isomorphic to $\mathfrak{t}_{g, r}^{n} \otimes \mathbb{C}$, to give a presentation of $\mathfrak{t}_{g, r}^{n} \otimes \mathbb{C}$, it suffices to give a presentation of its associated graded. It follows from Johnson's Theorem (7.1) that each graded quotient of the lower central series of $\mathfrak{t}_{g}$ is a representation of the algebraic group $S p_{g}$. We will give a presentation of $\mathrm{Gr}_{\bullet}^{W} \mathfrak{t}_{g}$ in the category of representations of $S p_{g}$. Recall that $\lambda_{1}, \ldots, \lambda_{g}$ is a set of fundamental weights of $S p_{g}$. For a nonnegative integral linear combination of the fundamental weights $\lambda=\sum_{i=1}^{g} n_{i} \lambda_{i}$ we denote by $V(\lambda)$ the representation of $S p_{g}$ with highest weight $\lambda$.

For all $g \geq 3$, the representation $\wedge^{2} V\left(\lambda_{3}\right)$ contains a unique copy of $V\left(2 \lambda_{2}\right)+$ $V(0)$. Denote the $S p_{g}$ invariant complement of this by $R_{g}$. Since the quadratic part of the free Lie algebra $\operatorname{Lie}(V)$ is $\wedge^{2} V$, we can view $R_{g}$ as being a subspace of the quadratic elements of $\operatorname{Lie}\left(V\left(\lambda_{3}\right)\right)$.

As mentioned earlier, it is unknown whether any $T_{g}$ is finitely presented when $g \geq 3$. But the following theorem says that its de Rham incarnation is:

Theorem 9.3. For all $g \geq 3, \mathfrak{t}_{g}$ is isomorphic to the completion of its associated graded $\mathrm{Gr}_{\bullet}^{W} \mathfrak{t}_{g}$. When $g \geq 6$, this has presentation

$$
\mathrm{Gr}_{\bullet}^{W} \mathfrak{t}_{g}=\operatorname{Lie}\left(V\left(\lambda_{3}\right)\right) /\left(R_{g}\right)
$$

where $R_{g}$ is the set of quadratic relations defined above. When $3 \leq g<6$, the relations in $\mathrm{Gr}^{W} \mathfrak{t}_{g}$ are generated by the quadratic relations $R_{g}$, and possibly some cubic relations. In particular, $t_{g, r}^{n}$ is finitely presented whenever $g \geq 3$.

Note that this, combined with (9.2) gives a presentation of $\mathrm{Gr}_{\bullet}^{W} \mathfrak{u}_{g}$ when $g \geq 6$ — just divide $\mathrm{Gr}_{\bullet}^{W} \mathfrak{t}_{g}$ by the unique quadratic central element to obtain $\operatorname{Gr}_{\bullet}^{W} \mathfrak{u}_{g}$. It also gives the following:

The proof that the relations in the presentation of $\mathfrak{t}_{g}$ are generated by quadratic relations when $g \geq 6$ and quadratic and cubic ones when $g \geq 3$ is not topological, but uses deep Hodge theory and, surprisingly, intersection homology. The key 
ingredients are a result of Kabanov which we state below, and M. Saito's theory of Hodge modules.

We define the Satake compactification $\overline{\mathcal{M}}_{g}^{\text {sat }}$ of $\mathcal{M}_{g}$ as the closure of $\mathcal{M}_{g}$ inside the (Baily-Borel-)Satake compactification of $\mathcal{A}_{g}$.

Theorem 9.4 (Kabanov [46]). For each irreducible representation $V$ of $S p_{g}$, the natural map

$$
I H^{2}\left(\overline{\mathcal{M}}_{g}^{\text {sat }} ; \mathbb{V}\right) \rightarrow H^{2}\left(\mathcal{M}_{g} ; \mathbb{V}\right)
$$

is an isomorphism when $g \geq 6$. Here $\mathbb{V}$ denotes the generically defined local system corresponding to $V$.

Such a local system $\mathbb{V}$ is, up to a Tate twist, canonically a variation of Hodge structure. Saito's purity theorem then implies that $H^{2}\left(\mathcal{M}_{g}, \mathbb{V}\right)$ is pure of weight $2+$ the weight of $\mathbb{V}$ when $g \geq 6$. It is this purity result that forces $H^{2}\left(\mathfrak{t}_{g}\right)$ to be of weight 2 , and implies that no higher order relations are needed.

9.3. Understanding $\mathfrak{t}_{g}$. Even though we have a presentation of $\mathfrak{t}_{g}$, we still do not have a good understanding of its graded quotients, either as vector spaces or as $S p_{g}$ modules. There is an exact sequence

$$
0 \rightarrow \mathfrak{p}_{g} \rightarrow \mathfrak{t}_{g}^{1} \rightarrow \mathfrak{t}_{g} \rightarrow 0
$$

of Lie algebras (recall that $\mathfrak{p}_{g}$ stands for the Malcev Lie algebra of $\pi_{g}=\pi_{1}\left(S, x_{0}\right)$ ).

It is the de Rham incarnation of the exact sequence of fundamental groups associated to the universal curve. Fix a conformal structure on $\left(S, x_{0}\right)$. Then this sequence is an exact sequence of MHSs. Since $\mathrm{Gr}^{W}$ is an exact functor, and since $\mathrm{Gr}_{\bullet}^{W} \mathfrak{p}_{g}$ is well understood, it suffices to understand $\mathrm{Gr}_{\bullet}^{W} \mathfrak{t}_{g}^{1}$.

There is a natural representation

$$
\mathfrak{t}_{g}^{1} \rightarrow \operatorname{Der} \mathfrak{p}_{g}
$$

It is a morphism of MHS, and therefore determined by the graded Lie algebra homomorphism

$$
\mathrm{Gr}_{\bullet}^{W} \mathfrak{t}_{g}^{1} \rightarrow \operatorname{Der} \mathrm{Gr}_{\bullet}^{W} \mathfrak{p}_{g}
$$

One can ask how close it is to being an isomorphism. Since this map is induced by the natural homomorphism

$$
\Gamma_{g}^{1} \rightarrow \lim _{\longleftarrow} \text { Aut } \mathbb{C} \pi_{g} / I^{m},
$$

the homomorphism (9.1) factors through the projection $\mathfrak{t}_{g}^{1} \rightarrow \mathfrak{u}_{g}^{1}$, and therefore cannot be injective. On the other hand, we have the following (reformulated) result of Morita:

Theorem 9.5 (Morita [68]). There is a natural Lie algebra surjection

$$
\operatorname{Tr}_{M}: W_{-1} \operatorname{Der~Gr}_{\bullet}^{W} \rightarrow \oplus_{k \geq 1} S^{2 k+1} H_{1}(S)
$$

onto an abelian Lie algebra whose composition with (9.1) is trivial. Here $S^{m}$ denotes the $m^{\text {th }}$ symmetric power.

One may then hope that the sequence

$$
0 \rightarrow \mathbb{C} \rightarrow \mathfrak{t}_{g}^{1} \rightarrow W_{-1} \operatorname{Der} \operatorname{Gr}_{\bullet}^{W} \mathfrak{p}_{g} \rightarrow \oplus_{k \geq 1} S^{2 k+1} H_{1}(S) \rightarrow 0
$$


is exact. However, there are further obstructions to exactness at $W_{-1} \operatorname{Der} \operatorname{Gr}_{\bullet}^{W} \mathfrak{p}_{g}$ which were discovered by Nakamura $[70]$. They come from Galois theory and use the fact that $\mathcal{M}_{g}^{1}$ is defined over $\mathbb{Q} \cdot \mathbb{T}^{\mathbb{T}}$ One the other hand, one can ask:

Question 9.6. Is the map $\mathfrak{u}_{g}^{1} \rightarrow \operatorname{Der}^{W} \mathfrak{p}_{g}$ injective? Equivalently, is $\mathcal{G}_{g}^{1}$ the Zariski closure of the image of the representation (9.2)?

A good understanding of $\mathfrak{t}_{g}$ may help in understanding the stable cohomology of $\Gamma_{g}$ as we shall explain in the next subsection.

9.4. Torelli Lie algebras and the cohomology of $\Gamma_{g}$. Each Malcev Lie algebra $\mathfrak{g}$ can be viewed as a complete topological Lie algebra. A basis for the neighbourhoods of 0 being the terms $\mathfrak{g}^{(k)}$ of the Malcev filtration. One can define the continuous cohomology of such a $\mathfrak{g}$ to be

$$
H^{\bullet}(\mathfrak{g}):=\underline{\lim _{\longrightarrow}} H^{\bullet}\left(\mathfrak{g} / \mathfrak{g}^{(k)}\right) .
$$

If $\mathfrak{g}$ has a MHS, then so will $H^{\bullet}(\mathfrak{g})$. The continuous cohomology of $\mathfrak{t}_{g, r}^{n}, \mathfrak{u}_{g, r}^{n}$, etc. each has an action of $S p_{g}$. The general theory of relative Malcev completion [25] gives a canonical homomorphism

$$
H^{\bullet}\left(\mathfrak{u}_{g, r}^{n}\right)^{S p_{g}} \rightarrow H^{\bullet}\left(\mathcal{M}_{g, r}^{n}\right)
$$

One can ask how much of the cohomology of $\mathcal{M}_{g, r}^{n}$ is captured by this map.

Fix a base point $x_{o}$ of $\mathcal{M}_{g, r}^{n}$. Then $\mathfrak{t}_{g, r}^{n}$, etc. all have compatible MHSs, and these induce MHSs on their continuous cohomology groups. These groups have the property that the weights on $H^{k}$ are $\geq k$.

Theorem 9.7 (Hain [26). The map (9.3) is a MHS morphism.

Since $H_{1}\left(T_{g}\right)$ is a quotient of $\mathfrak{u}_{g}$, there is an induced map

$$
H^{\bullet}\left(H_{1}\left(T_{g}\right)\right) \rightarrow H^{\bullet}\left(\mathfrak{u}_{g}\right) .
$$

This is also a morphism of MHS. The following result follows directly from 22 , (9.2)], the presentation (9.3) of $\mathfrak{t}_{g}$, and the existence of the MHS on $\mathfrak{u}_{g}$.

Proposition 9.8. If $g \geq 3$, the map (9.4) surjects onto the lowest weight subring

$$
\oplus_{k \geq 0} W_{k} H^{k}\left(\mathfrak{u}_{g}\right)
$$

of $H^{\bullet}\left(\mathfrak{u}_{g}\right)$, and the kernel is generated by the ideal generated by the unique copy of $V\left(2 \lambda_{2}\right)$ in $H^{2}\left(H_{1}\left(T_{g}\right)\right)$.

Similar results hold when $r+n>0$ - cf. [26, §14.6].

The following result of Kawazumi and Morita tells us that the image of the lowest weight subring of $H^{\bullet}\left(\mathfrak{u}_{g}\right)^{S p_{g}}$ contains no new cohomology classes.

Theorem 9.9 (Kawazumi-Morita 48]). The image of the natural map

$$
H^{\bullet}\left(H_{1}\left(T_{g}\right)\right)^{S p_{g}} \rightarrow H^{\bullet}\left(\mathcal{M}_{g}\right)
$$

is precisely the subring generated by the $\kappa_{i}$ 's.

If we combine this with the previous two results and Pikaart's Purity Theorem (4.2), we obtain the following strengthening of the theorem of Kawazumi and Morita.

\footnotetext{
${ }^{11}$ Actually, Nakamura proves his result for a corresponding sequence for $\mathfrak{t}_{g, 1}$, but his obstructions most likely appear in this case too.
} 
Theorem 9.10. When $k \leq g / 2$, the image of $H^{\bullet}\left(\mathfrak{u}_{g}\right)^{S p_{g}} \rightarrow H^{\bullet}\left(\mathcal{M}_{g}\right)$ is the subring generated by the $\kappa_{i}$ 's.

To continue the discussion further, it seems useful to consider cohomology with symplectic coefficients.

9.5. Cohomology with symplectic coefficients. The irreducible representations of $S p_{g}$ are parametrized by Young diagrams with $\leq g$ rows (and no indexing of the boxes), in other words by nonincreasing sequences of nonnegative integers whose terms with index $>g$ are zero. So any such sequence $\alpha=\left(\alpha_{1}, \alpha_{2}, \ldots\right)$ defines an irreducible representation of $S p_{h}$ for all $h \geq g$. We will denote the representation of $S p_{g}$ corresponding to $\alpha$ by $V_{g, \alpha}$, and the corresponding (orbifold) local system over $\mathcal{M}_{g}$ by $\mathbb{V}_{g, \alpha}$.

A theorem of Ivanov [36] (that in fact pertains to more general local systems) implies that, when $r \geq 1$, the group $H^{k}\left(\Gamma_{g, r}^{n}, V_{g, \alpha}\right)$ is independent of $g$ once $g$ is large enough. In the case at hand we have a more explicit result that we state here for the undecorated case (a case that Ivanov actually excludes).

Theorem 9.11 (Looijenga [58]). Let $\alpha=\left(\alpha_{1}, \alpha_{2}, \ldots\right)$ be a nonincreasing sequence of nonnegative integers that is eventually zero, and let $c_{1}, c_{2}, \ldots$ be weighted variables with $\operatorname{deg}\left(c_{i}\right)=2 i$. Put $|\alpha|:=\sum_{i \geq 1} \alpha_{i}$. Then there exists a finitely generated, evenly graded $\mathbb{Q}\left[c_{1}, \ldots, c_{|\alpha|}\right]$-module $A_{\alpha}^{\bullet}$ (that can be described explicitly) and a graded homomorphism of $H^{\bullet}\left(\Gamma_{\infty}\right)$ modules

$$
A_{\alpha}^{\bullet}[-|\alpha|] \otimes H^{\bullet}\left(\Gamma_{\infty}\right) \rightarrow H^{\bullet}\left(\Gamma_{g}, V_{g, \alpha}\right)
$$

that is an isomorphism in degree $\leq c g-|\alpha|$. It is also a MHS morphism if we take $A_{\alpha}^{2 k}$ to be pure of type $(k, k)$. In particular, we have

$$
A_{\alpha}^{\bullet}[-|\alpha|] \otimes H^{\bullet}\left(\Gamma_{\infty}\right) \cong H^{\bullet}\left(\Gamma_{\infty}, V_{\alpha}\right)
$$

both as MHSs and as graded $H^{\bullet}\left(\Gamma_{\infty}\right)$ modules. So, by (4.F), $H^{k}\left(\Gamma_{\infty}, V_{\alpha}\right)$ is pure of weight $k+|\alpha|$.

It is useful to try to understand all cohomology groups with symplectic coefficients at the same time. To do this we take a leaf out of the physicist's book and consider the "generating function"

$$
\oplus_{\alpha} H^{\bullet}\left(\Gamma_{g}, V_{\alpha}^{*}\right) \otimes V_{\alpha}
$$

where $\alpha$ ranges over all partitions with $\leq g$ rows, and $*$ denotes dual. This is actually a graded commutative ring as the Peter-Weyl Theorem implies that the coordinate ring $\mathcal{O}_{g}$ of $S p_{g}$ is

$$
\mathcal{O}_{g}=\oplus_{\alpha} \text { End } V_{\alpha} \cong \oplus_{\alpha} V_{\alpha}^{*} \otimes V_{\alpha} .
$$

The mapping class group acts on $\mathcal{O}_{g}$ by composing the right translation action of $S p_{g}$ on $\mathcal{O}_{g}$ with the canonical representation $\Gamma_{g} \rightarrow S p_{g}$. The corresponding cohomology group $H^{\bullet}\left(\Gamma_{g}, \mathcal{O}\right)$ is then the "generating function" (9.5). Note that $\mathcal{O}_{g}$ is a variation of Hodge structure of weight 0 , so the group $H^{k}\left(\overline{\Gamma_{g}}, \mathcal{O}_{g}\right)$ is stably of weight $k$ by the above theorem.

There is a canonical algebra homomorphism

$$
H^{\bullet}\left(\mathfrak{u}_{g}\right) \rightarrow H^{\bullet}\left(\Gamma_{g}, \mathcal{O}_{g}\right)
$$

whose existence follows from the de Rham theory of relative completion suggested by Deligne - cf. 25]. The map (9.3) of the previous subsection is just its invariant 
part. This map is a MHS morphism for each choice of complex structure on $S$. The $\alpha$ isotypical part of both sides stabilizes as $g$ increases. It is natural to ask:

Question 9.12. Is this map stably an isomorphism?

This has been verified by Hain and Kabanov (unpublished) in degrees $\leq 3$. If the answer is yes, or even if one has surjectivity, then it will follow from the theorem of Kawazumi and Morita (9.9) that the stable cohomology of $\mathcal{M}_{g}$ is generated by the $\kappa_{i}$ 's. A consequence of injectivity and Pikaart's Purity Theorem would be that for each $k, H^{k}\left(\mathfrak{u}_{g}\right)$ is pure of weight $k$ once the genus is sufficiently large. This is equivalent to the answer to the following question being affirmative.

Question 9.13. Are $H^{\bullet}\left(\mathfrak{u}_{g}\right)$ and $U \mathrm{Gr}_{\bullet}^{W} \mathfrak{u}_{g}$ are stably Koszul dual?

Note that the lowest weight subalgebra of $H^{\bullet}\left(\mathfrak{u}_{g}\right)$ and $U \mathrm{Gr}_{\bullet}^{W} \mathfrak{u}_{g}$ have dual quadratic presentations.

\section{Algebras Related to the Cohomology of Moduli Spaces of Curves}

The ribbon graph description is the root of a number of ways of constructing (co)homology classes on moduli spaces of curves from certain algebraic structures. These constructions have in common that they actually produce cellular (co)chains on $\mathbb{M}_{g}^{n}$, and so they are recipes that assign numbers to 'oriented' ribbon graphs. The typical construction, due to Kontsevich [52], goes like this: assume that we are given a complex vector space $V$, a symmetric tensor $p \in V \otimes V$, and linear forms $T_{k}: V^{\otimes k} \rightarrow \mathbb{C}$ that are cyclically invariant. If $\Gamma$ is an ribbon graph, then the decomposition of $X(G)$ into $\sigma_{1}$ and $\sigma_{o}$-orbits gives isomorphisms

$$
\otimes_{s \in X_{1}(\Gamma)} V^{\otimes \text { or }(s)} \cong V^{\otimes X(G)} \cong \otimes_{v \in X_{0}(\Gamma)} V^{\otimes \text { out }(v)},
$$

where or $(s)$ stands for the two-element set of orientations of the edge $s$ and out $(v)$ for the set of oriented edges that have $v$ as initial vertex. Now $p^{\otimes X_{1}(\Gamma)}$ defines a vector of the lefthand side and a tensor product of certain $T_{k}$ 's defines a linear form on the righthand side. Evaluation of the linear form on the vector gives a number, which is clearly an invariant of the ribbon graph. Since this invariant does not depend on an orientation on the set of edges of $G$, it cannot be used directly to define a cochain on the combinatorial moduli spaces. To this end we need some sign rules so that, for instance, the displayed isomorphisms acquire a sign. The tensors $p$ and $T_{k}$ are sometimes referred to as the propagator and the interactions, respectively, to remind us of their physical origin.

If $p$ is nondegenerate, then we may use it to identify $V$ with its dual. In this case $T_{k}$ defines a linear map $V^{\otimes(k-1)} \rightarrow V$. The properties one needs to impose on propagator and interactions in order that the above recipe produce a cocycle on $\mathbb{M}_{g}^{n}$ is that they define a $\mathbb{Z} / 2$ graded $A_{\infty}$ algebra with inner product. A similar recipe assigns cycles on $\mathbb{M}_{g}^{n}$ to certain $\mathbb{Z} / 2$ graded differential algebras. The cocycles can be evaluated on the cycles and this, in principle, gives a method of showing that some of the classes thus obtained are nonzero. We shall not be more precise, but instead refer to 52] or [1] for an overview. A simple example is to take $V=\mathbb{C}$, $p:=1 \otimes 1$ and $T_{k}\left(z^{k}\right)$ arbitrary for $k \geq 3$ odd, and zero otherwise. Kontsevich asserts that the classes thus obtained are all tautological. 
10.1. Outer space. In Section 6 we encountered a beautiful combinatorial model for a virtual classifying space of the mapping class group $\Gamma_{g}^{n}$. There is a similar, but simpler, combinatorial model that does the same job for the outer automorphism group of a free group.

We fix an integer $r \geq 2$ and consider connected graphs $G$ with first Betti number equal to $r$ and where each vertex has degree $\geq 3$. Let us call these graphs $r$ circular graphs. The maximal number of edges (resp. vertices) such a graph can have is $3 g-3$ (resp. $2 r-2$ ). These bounds are realized by all trivalent graphs of this type. Notice that an $r$-circular graph $G$ has fundamental group isomorphic to the free group on $r$ generators, $F_{r}$. We say that $G$ is marked if we are given an isomorphism $\phi: F_{r} \rightarrow \pi_{1}(G$, base point) up to inner automorphism. The group $\operatorname{Out}\left(F_{r}\right)$ permutes these markings simply transitively. There is an obvious notion of isomorphism for marked $r$-circular graphs. We shall denote the collection of isomorphism classes by $\mathcal{G}_{r}$.

Let $(G,[\phi])$ represent an element of $\mathcal{G}_{r}$. The metrics on $G$ that give $G$ total length 1 are parameterized by the interior of a simplex $\Delta(G)$. We fit these simplices together in a way analogous to the ribbon graph case: if $s$ is an edge of $G$ that is not a loop, then collapsing it defines another element $(G / s,[\phi] / s)$ of $\mathcal{G}_{r}$. We may then identify $\Delta(G / s)$ with a face of $\Delta(G)$. After we have made these identifications we end up with a simplicial complex $\widehat{\mathbb{O}}_{r}$. The union of the interiors of the simplices $\Delta(G)$ (indexed by $\mathcal{G}_{r}$ ) will be denoted by $\mathbb{O}_{r}$; it is the complement of a closed subcomplex of $\widehat{\mathbb{O}}_{r}$. This construction is due to Culler-Vogtmann [9]. We call $\mathbb{O}_{r}$ the outer space of order $r$ for reasons that will become apparent in a moment. Observe that $\widehat{\mathbb{O}}_{r}$ comes with a simplicial action of $\operatorname{Out}\left(F_{r}\right)$. We denote the quotient of $\widehat{\mathbb{O}}_{r}\left(\right.$ resp. $\left.\mathbb{O}_{r}\right)$ by $\operatorname{Out}\left(F_{r}\right)$ by $\widehat{\mathbb{G}}_{r}\left(\right.$ resp. $\left.\mathbb{G}_{r}\right)$. It is easy to see that $\widehat{\mathbb{G}}_{r}$ is a finite orbicomplex. The open subset $\mathbb{G}_{r}$ is the moduli space of metrized $r$-circular graphs. It has a spine of dimension $2 r-3$.

Theorem 10.1 (Culler-Vogtman [9], Gersten). The Outer space of order $r$ is contractible and a subgroup of finite index of $\operatorname{Out}\left(F_{r}\right)$ acts freely on it. Hence $\mathbb{G}_{r}$ is a virtual classifying space for $\operatorname{Out}\left(F_{r}\right)$ and $\operatorname{Out}\left(F_{r}\right)$ has virtual homological dimension $\leq 2 r-3$.

In contrast to the ribbon graph case, $\mathbb{O}_{r}$ is not piecewise smooth. If we choose $2 g-1+n$ free generators for the fundamental group of our reference surface $S_{g}^{n}$, then each ribbon graph without vertices of degree $\leq 2$ determines an element of $\mathbb{G}_{2 g-1+n}$ : simply forget the ribbon structure. The ribbon data is finite and it is therefore not surprising that forgetting the ribbons defines a finite map

$$
\widehat{\text { fr }}: \mathcal{S}_{n} \backslash \widehat{\mathbb{M}}_{g}^{n} \rightarrow \widehat{\mathbb{G}}_{2 g-1+n}
$$

of orbicomplexes. Here $\mathcal{S}_{n}$ stands for the symmetric group, which acts in the obvious way on $\widehat{\mathbb{M}}_{g}^{n}$. Following Strebel's theorem, the preimage of $\mathbb{G}_{2 g-1+n}$ can be identified with $\mathcal{S}_{n} \backslash\left(\mathcal{M}_{g}^{n} \times\right.$ int $\left.\Delta^{n-1}\right)$. We denote the resulting map by

$$
\text { fr : } \mathcal{S}_{n} \backslash\left(\mathcal{M}_{g}^{n} \times \operatorname{int} \Delta^{n-1}\right) \rightarrow \mathbb{G}_{2 g-1+n} .
$$

It induces the evident map

$$
\mathrm{fr}_{*}: H_{k}\left(\Gamma_{g}^{n}\right)_{\mathcal{S}_{n}} \rightarrow H_{k}\left(\mathrm{Out} F_{2 g-1+n}\right)
$$

on rational homology. It is unclear whether there is such an interpretation for the induced map on cohomology with compact supports. We remark that $\mathcal{M}_{g}^{n} \times \operatorname{int} \Delta^{n-1}$ 
is canonically oriented, but that its $\mathcal{S}_{n}$-orbit space is not (since transpositions reverse this orientation). Poincaré duality therefore takes the form

$$
H_{k}\left(\mathcal{M}_{g}^{n}\right)_{\mathcal{S}_{n}} \cong H_{k}\left(\mathcal{S}_{n} \backslash\left(\mathcal{M}_{g}^{n} \times \operatorname{int} \Delta^{n-1}\right) ; \epsilon\right) \cong H_{c}^{6 g-7+3 n-k}\left(\mathcal{S}_{n} \backslash\left(\mathcal{M}_{g}^{n} \times \operatorname{int} \Delta^{n-1}\right) ; \epsilon\right),
$$

where $\epsilon$ is the signum representation of $\mathcal{S}_{n}$. If $\delta$ denotes the (signum) character of $\operatorname{Out}\left(F_{r}\right)$ on $\wedge^{r} H_{1}\left(F_{r}\right)$, then the adjoint of $\mathrm{fr}_{*}$ is a map

$$
H_{c}^{6 g-7+3 n-k}\left(\mathbb{G}_{2 g-1+n} ; \delta\right) \rightarrow H_{c}^{6 g-7+3 n-k}\left(\mathcal{S}_{n} \backslash\left(\mathcal{M}_{g}^{n} \times \operatorname{int} \Delta^{n-1}\right) ; \epsilon\right) \cong H_{k}\left(\mathcal{M}_{g}^{n}\right)_{\mathcal{S}_{n}} .
$$

So, when $m \geq 1$, we have maps

$$
H_{c}^{i+m-1}\left(\mathbb{G}_{m+1} ; \delta\right) \rightarrow \oplus_{2 g-2+n=m} H_{2 m-i}\left(\mathcal{M}_{g}^{n}\right)_{\mathcal{S}_{n}} \rightarrow H_{2 m-i}\left(\mathbb{G}_{m+1}\right), \quad i=0,1, \ldots
$$

There is a remarkable interpretation of this sequence that we will discuss next.

10.2. Three Lie algebras. We describe Kontsevich's three functors from the category of symplectic vector spaces to the category of Lie algebras and their relation with the cohomology of the moduli spaces $\mathcal{M}_{g}^{n}$. The basic references are [51] and 52 .

We start out with a finite dimensional $\mathbb{Q}$ vector space $V$ endowed with a nondegenerate antisymmetric tensor $\omega_{V} \in V \otimes V$. Let $\operatorname{Ass}(V)$ be the tensor algebra (i.e., the free associative algebra) generated by $V$. We grade it by giving $V$ degree -1 . The Lie subalgebra generated by $V$ is free and so we denote it by $\operatorname{Lie}(V)$. It is well-known that $\operatorname{Ass}(V)$ may be identified with the universal enveloping algebra of $\operatorname{Lie}(V)$. If we mod out $\operatorname{Ass}(V)$ by the two-sided ideal generated by the degree $\leq-2$ part of $\operatorname{Lie}(V)$, we obtain the symmetric algebra $\operatorname{Com}(V)$ of $V$. Define $\mathfrak{g}_{\text {ass }}(V)$ (resp. $\mathfrak{g}_{\text {lie }}(V)$ ) to be the Lie algebra of derivations of $\operatorname{Ass}(V)$ (resp. Lie $(V)$ ) of degree $\leq 0$ that kill $\omega_{V}$. Since each derivation of $\operatorname{Lie}(V)$ extends canonically to its universal enveloping algebra, we have an inclusion $\mathfrak{g}_{\text {lie }}(V) \subset \mathfrak{g}_{\text {ass }}(V)$. There is also a corresponding Lie algebra $\mathfrak{g}_{\text {com }}(V)$ of derivations of degree $\leq 0$ of $\operatorname{Com}(V)$ that kill $\omega_{V}$. Here we regard the latter as a two-form on the affine space $\operatorname{Spec} \operatorname{Com}(V)$. This Lie algebra is a quotient of $\mathfrak{g}_{\text {ass }}(V)$. All three Lie algebras are graded and have as degree zero summand the Lie algebra $\mathfrak{s p}(V)$ of the group $\operatorname{Sp}(V)$ of symplectic transformations of $V$. A simple verification shows that the degree -1 summands have as $\mathfrak{s p}(V)$ representations the following natural descriptions:

$$
\mathfrak{g}_{\text {com }}(V)_{-1} \cong S^{3}(V), \quad \mathfrak{g}_{\text {ass }}(V)_{-1} \cong S^{3}(V) \oplus \wedge^{3} V, \quad \mathfrak{g}_{\text {lie }}(V)_{-1} \cong \wedge^{3} V .
$$

These Lie algebras themselves do not behave functorially with respect to morphisms $\left(V, \omega_{V}\right) \rightarrow\left(W, \omega_{W}\right)$, but their cohomology does. To see this, just notice that $\operatorname{Sp}(V)$ acts trivially on this cohomology of the Lie algebra in question because $\mathfrak{s p}(V) \subset \mathfrak{g}_{*}(V)$. This implies that $H^{\bullet}\left(\mathfrak{g}_{*}(V)\right)$ depends only on $\operatorname{dim} V$. Any morphism $\left(V, \omega_{V}\right) \rightarrow\left(W, \omega_{W}\right)$ is the projection onto a direct summand and so induces a map $H^{\bullet}\left(\mathfrak{g}_{*}(W)\right) \rightarrow H^{\bullet}\left(\mathfrak{g}_{*}(V)\right)$. We form the direct limit:

$$
H^{\bullet}\left(\mathfrak{g}_{*}\right):=\varliminf_{\longrightarrow} H^{\bullet}\left(\mathfrak{g}_{*}(V)\right) .
$$

This has the structure of a connected graded bicommutative Hopf algebra; the coproduct comes from the direct sum operation on symplectic vector spaces. It is actually bigraded: apart from the cohomological grading there is another coming from grading of the Lie algebras. Notice that the latter grading has all its degrees $\geq 0$. The primitive part $H_{\mathrm{pr}}^{\bullet}\left(\mathfrak{g}_{*}\right)$ inherits this bigrading. Furthermore, the natural maps

$$
H^{\bullet}\left(\mathfrak{g}_{\mathrm{com}}\right) \rightarrow H^{\bullet}\left(\mathfrak{g}_{\mathrm{ass}}\right) \rightarrow H^{\bullet}\left(\mathfrak{g}_{\mathrm{lie}}\right)
$$


are homomorphisms of bigraded Hopf algebras. Consequently, we have induced maps between the bigraded pieces of their primitive parts.

Theorem 10.2 (Kontsevich 51, [52]). We have

$$
H_{\mathrm{pr}}^{k}\left(\mathfrak{g}_{*}\right)_{0}=H_{\mathrm{pr}}^{k}\left(\mathfrak{s p}_{\infty}\right) \cong \begin{cases}\mathbb{Q} & \text { for } k=3,7,11, \ldots ; \\ 0 & \text { otherwise }\end{cases}
$$

Furthermore, $H_{\mathrm{pr}}^{k}\left(\mathfrak{g}_{*}\right)_{l}=0$ when $l$ is odd and, when $m>0$, we have natural diagram

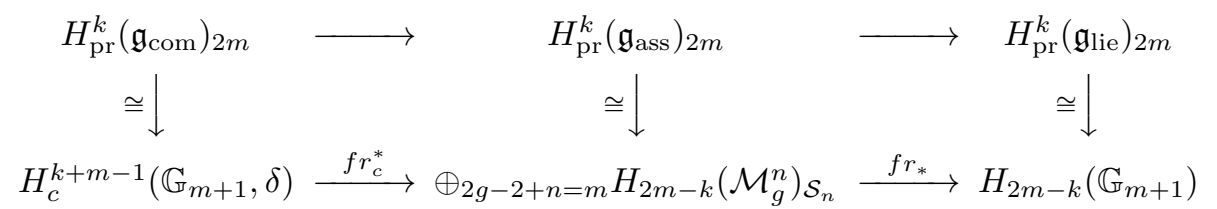

which commutes up to sign and whose rows are complexes. The maps in the top row are the natural maps and the bottom row is the sequence defined in Subsection 10.1.

The proof is an intelligent application of classical invariant theory. For each of the three Lie algebras one writes down the standard complex. The subcomplex of invariants with respect to the symplectic group is quasi-isomorphic to the full complex. Weyl's invariant theory furnishes a natural basis for this subcomplex. Kontsevich then observes that this makes the subcomplex naturally isomorphic to a cellular chain (or cochain) complex of one of the cell complexes $\mathbb{G}_{*}$ and $\mathbb{M}_{*}^{*}$ whose (co)homology appears in the bottom row.

The diagram in this theorem suggests that the sequence of natural transformations Lie $\rightarrow$ Ass $\rightarrow$ Com is self dual in some sense. This can actually be pinned down by looking at the corresponding operads: Ginzburg and Kapranov [18 observed that these operads have "quadratic relations" and they proved the self duality of the operad sequence in a Koszul sense.

However, our main reason for displaying this diagram is that it pertains to the cohomology of the moduli spaces of curves in two apparently unrelated ways. The first one is evident. The $\mathcal{S}_{n}$ coinvariants of the homology of $\mathcal{M}_{g}^{n}$ features in the middle column, but the righthand column has something to do with the cohomology of a 'linearization' of $\Gamma_{\infty}$ : we will see that $\mathfrak{g}_{\text {lie }}$ is intimately related to the Lie algebra of the relative Malcev completions discussed in Section 8. We explain this in the next subsection.

10.3. Relation with the relative Malcev completion. We begin with making an observation. For a symplectic vector space $V$, let $\mathfrak{g}_{*}^{b}(V)$ be the subalgebra of $\mathfrak{g}_{*}(V)$ generated by the summands of weight 0 and -1 . Kontsevich's computation shows:

Proposition 10.3. The graded cohomology groups $H^{k}\left(\mathfrak{g}_{*}^{b}(V)\right)_{l}$ stabilize, their stable limit is a bigraded bicommutative Hopf algebra $H^{\bullet}\left(\mathfrak{g}_{*}^{b}\right)$. In addition, the restriction map $H_{\mathrm{pr}}^{k}\left(\mathfrak{g}_{*}\right)_{l} \rightarrow H_{\mathrm{pr}}^{k}\left(\mathfrak{g}_{*}^{b}\right)_{l}$ is an isomorphism when $l \leq k$.

The case of interest is here is that of Lie, where $\mathfrak{g}_{\text {lie }}^{b}(V)_{0}=\mathfrak{s p}(V)$ and $\mathfrak{g}_{\text {lie }}^{b}(V)_{-1} \cong$ $\wedge^{3} V$. If $z_{m} \in H_{\mathrm{pr}}^{2 m}\left(\mathfrak{g}_{\text {lie }}\right)_{2 m}$ corresponds via theorem 10.2 to $1 \in H_{0}\left(\mathbb{G}_{m+1}\right)$, then the preceding proposition yields: 
Corollary 10.4. We have a natural isomorphism of bigraded Hopf algebras

$$
\sum_{l \leq k} H^{k}\left(\mathfrak{g}_{\mathrm{lie}}^{b}\right)_{l} \cong H^{\bullet}\left(\mathfrak{s p}_{\infty}\right)\left[z_{1}, z_{2}, \ldots\right]
$$

where $H^{\bullet}\left(\mathfrak{s p}_{\infty}\right)$ has weight zero.

Next we relate the graded Lie algebra $\mathfrak{g}_{\text {lie }}^{b}$ to the filtered Lie algebra $\mathfrak{g}_{g, 1}$ of the relative Malcev completion $\mathcal{G}_{g, 1}$ of $\Gamma_{g, 1} \rightarrow S p_{g}$. Recall from Section 2 that $\pi_{g}^{1}$ is freely generated by $2 g$ generators named $\alpha_{ \pm}, \ldots, \alpha_{ \pm g}$ so that the commutator $\beta:=\left(\alpha_{1}, \alpha_{-1}\right) \cdots\left(\alpha_{g}, \alpha_{-g}\right)$ represents a simple loop around $x_{1}$. Using Latin letters for the logarithms of the images of elements of $\pi_{g}^{1}$ in its Malcev completion, we find that

$$
b \equiv\left[a_{1}, a_{-1}\right]+\cdots+\left[a_{g}, a_{-g}\right] \quad\left(\bmod \left(\mathfrak{p}_{g}^{1}\right)^{(3)}\right) .
$$

So the image of $b$ in $\operatorname{Gr}^{2} \mathfrak{p}_{g}^{1} \cong \wedge^{2} V_{g}$ is the cup symplectic form $\omega_{S}$.

The obvious homomorphism $\Gamma_{g, 1} \rightarrow \operatorname{Aut}\left(\pi_{g}^{1}\right)$ induces a Lie algebra homomorphism

$$
\mathfrak{g}_{g, 1} \rightarrow \text { Der } \mathfrak{p}_{g}^{1} .
$$

whose image we denote by $\overline{\mathfrak{g}}_{g, 1}$. (It is possible that this map is injective so that $\left.\mathfrak{g}_{g, 1} \cong \overline{\mathfrak{g}}_{g, 1} \cdot\right)$

Notice that $\overline{\mathfrak{g}}_{g, 1}$ is contained in the subalgebra $\operatorname{Der}\left(\mathfrak{p}_{g, 1}, b\right)$ consisting of those derivations that kill $b$. Since (10.1) is (Malcev) filtration preserving, it induces Lie algebra homomorphisms

$$
\operatorname{Gr}^{\bullet} \mathfrak{g}_{g, 1} \rightarrow \operatorname{Gr}^{\bullet} \overline{\mathfrak{g}}_{g, 1} \rightarrow \operatorname{Der}\left(\operatorname{Gr}^{1}, \omega_{S}\right) .
$$

Notice that the last term is just $\mathfrak{g}_{\text {lie }}\left(V_{g}\right)$.

At this stage we need Hodge theory. Choose a conformal structure on $S_{g}$. Then, by (9.1), there are natural MHSs on $\mathfrak{g}_{g, 1}$ and $\mathfrak{p}_{g, 1}$ whose weight filtrations are the Malcev filtrations and such that (10.1) is a MHS morphism. Hence the image $\overline{\mathfrak{g}}_{g, 1}$ has a natural MHS. Since $\mathrm{Gr}^{\bullet} \mathfrak{g}_{g, 1}$ is generated by its summands in degree 0 and 1 , the same is true for $\mathrm{Gr}^{\bullet} \overline{\mathfrak{g}}_{g, 1}$. On the other hand, the summands in degree 0 and 1 of $\operatorname{Gr}^{\bullet} \overline{\mathfrak{g}}_{g, 1}$ are equal to the summands of weight 0 and -1 of $\mathfrak{g}_{\text {lie }}\left(V_{g}\right)$, and so the graded Lie algebra $\operatorname{Gr}^{\bullet} \overline{\mathfrak{g}}_{g, 1}$ may be identified with $\mathfrak{g}_{\text {lie }}^{b}\left(V_{g}\right) \bullet$ (except that the indexing of the summands differs by sign).

We know from Section 9 that the homomorphisms

$$
H^{\bullet}\left(\overline{\mathfrak{g}}_{g, 1}\right) \rightarrow H^{\bullet}\left(\mathfrak{g}_{g, 1}\right) \rightarrow H^{\bullet}\left(\Gamma_{g, 1}\right)
$$

are morphisms of MHS. After weight grading these become bigraded algebra homomorphisms

$$
H^{\bullet}\left(g_{\text {lie }}^{b}\left(V_{g}\right) \bullet\right) \rightarrow H^{\bullet}\left(\mathrm{Gr}_{\bullet}^{W} \mathfrak{g}_{g, 1}\right) \rightarrow \mathrm{Gr}_{\bullet}^{W} H^{\bullet}\left(\Gamma_{g, 1}\right) .
$$

The sequence 10.2 stabilizes with $g$ to a sequence of Hopf algebras in the MHS category. The corresponding weight graded sequence is

$$
H^{\bullet}\left(\left(g_{\mathrm{lie}}^{\mathrm{b}}\right) \bullet\right) \rightarrow H^{\bullet}\left(\mathrm{Gr}_{\bullet}^{W} \mathfrak{g}_{\infty}\right) \rightarrow \mathrm{Gr}_{\bullet}^{W} H^{\bullet}\left(\Gamma_{\infty}\right) .
$$

But by Pikaart's Purity Theorem we know that the last term is pure of weight $k$ in degree $k$, so that we can replace it by $H^{\bullet}\left(\Gamma_{\infty}\right)$. If we compose the natural restriction map $H^{\bullet}\left(g_{\text {lie }}\right) \rightarrow H^{\bullet}\left(g_{\text {lie }}^{\text {b }}\right)$ with the above maps we get a homorphism

$$
H^{\bullet}\left(g_{\text {lie }}\right) \rightarrow H^{\bullet}\left(\Gamma_{\infty}\right) \text {. }
$$


Kontsevich asked (at the end of 52 ) about the meaning of this map. This can now be answered by invoking the theorem of Kawazumi and Morita (9.9), or rather a weaker form, which says that $z_{i}$ is mapped to a nonzero multiple of $\kappa_{i}$ :

Theorem 10.5. Let $\overline{\mathcal{G}}_{g, 1}$ denote the Zariski closure of $\Gamma_{g, 1}$ in Aut $\left(\mathfrak{p}_{q}^{1}\right)$ and $\overline{\mathfrak{g}}_{g, 1}$ its Lie algebra. Then the continuous cohomology of $\overline{\mathfrak{g}}_{g, 1}$ has a natural MHS such that $H^{\bullet}\left(\overline{\mathfrak{g}}_{g, 1}\right) \rightarrow H^{\bullet}\left(\Gamma_{g, 1}\right)$ is a MHS morphism. All this stabilizes with $g$ and results in a Hopf algebra $H^{\bullet}\left(\overline{\mathfrak{g}}_{\infty}\right)$ with a MHS and a Hopf algebra homomorphism

$$
H^{\bullet}\left(\overline{\mathfrak{g}}_{\infty}\right) \rightarrow H^{\bullet}\left(\Gamma_{\infty}\right)
$$

that is at the same time a MHS morphism. Its image is $\mathbb{Q}\left[\kappa_{1}, \kappa_{2}, \ldots\right]$ and its kernel the ideal generated by the primitive classes whose weight differs from its degree.

Likewise, we have a natural Hopf algebra homomorphism

$$
H^{\bullet}\left(\mathfrak{g}_{\text {lie }}\right) \rightarrow H^{\bullet}\left(\Gamma_{\infty}\right)
$$

with the same image $\mathbb{Q}\left[\kappa_{1}, \kappa_{2}, \ldots\right]$ and the same description of its kernel.

Recent work of Kawazumi and Morita attempts to explain the kernel of the homomorphism $H^{\bullet}\left(\overline{\mathfrak{g}}_{\infty}\right) \rightarrow H^{\bullet}\left(\Gamma_{\infty}\right)$. In the present setting this might begin with the observation that the stable cohomology of the symplectic group appears here in an artificial way and simply disappears if we are more careful. As we shall see, this happens at the cost of introducing other primitive classes, but these turn out to be more geometric. The idea is to replace the Lie algebra cohomology of $\overline{\mathfrak{g}}_{g, 1}$ by the relative Lie algebra cohomology of the pair $\left(\overline{\mathfrak{g}}_{g, 1}, \mathfrak{k}_{g}\right)$, where $\mathfrak{k}_{g}$ is a maximal compact Lie subalgebra of $\overline{\mathfrak{g}}_{g, 1}$ (a unitary Lie algebra of rank $g$ ). This is more natural, because the Lie algebra complex of this pair can be understood as the de Rham complex of translation invariant forms on a classifying space for MHSs on $\mathfrak{p}_{g, 1}$. We then have a map on cohomology $H^{\bullet}\left(\overline{\mathfrak{g}}_{g, 1}, \mathfrak{k}_{g}\right) \rightarrow H^{\bullet}\left(\mathcal{M}_{g, 1}\right)$ with a geometric origin: it is induced by a period map from $\mathcal{M}_{g, 1}$ to this classifying space. The stable cohomology $H^{\bullet}\left(\overline{\mathfrak{g}}_{g, 1}, \mathfrak{k}_{g}\right)$ is now the same as that of $H^{\bullet}\left(\overline{\mathfrak{g}}_{g, 1}\right)$, except that the tensor factor $H^{\bullet}\left(\mathfrak{s p}_{\infty}\right)$ gets replaced by the stable cohomology of $\left(\mathfrak{s p}_{g}, \mathfrak{k}_{g}\right.$ ) (or equivalently, of $\mathcal{A}_{g}$ ). The latter is simply the polynomial algebra on the odd universal Chern classes $\lambda_{1}, \lambda_{3}, \lambda_{5}, \cdots$. These classes do not map to zero in $H^{\bullet}\left(\mathcal{M}_{g, 1}\right)$, but according to Mumford [69], they map to polynomials in the odd $\kappa$ 's: $\kappa_{1}, \kappa_{3}, \kappa_{5}, \ldots$. This indicates that no new stable classes are to be expected from Hodge theory, that is, a de Rham version of the Mumford conjecture holds.

\section{REFERENCES}

[1] Amsterdam-Utrecht seminar on moduli spaces of curves, C. Faber and E. Looijenga, editors, A. and K. Peters Inc., to appear.

[2] E. Arbarello, M. Cornalba, P. Griffiths and J. Harris: Geometry of Algebraic Curves I Grundlehren Math. Wiss. 267, Springer Verlag (1985).

[3] E. Arbarello and M. Cornalba: The Picard group of the moduli space of curves, Topology 26 (1987), 153-171.

[4] E. Arbarello and M. Cornalba: Combinatorial and algebro-geometric cohomology classes on the moduli spaces of curves, to appear in the J. of Alg. Geom.

[5] A. Borel: Stable real cohomology of arithmetic groups, Ann. Sci. Ecole Norm. Sup. 7 (1974), 235-272.

[6] A. Borel: Stable real cohomology of arithmetic groups II, Manifolds and Groups, Papers in Honor of Yozo Matsushima, Progress in Mathematics 14 , Birkhauser, Boston, 1981, 21-55.

[7] J. Carlson: The geometry of the extension class of a mixed Hodge structure, in Algebraic Geometry Bowdoin, 1985, Proc. Symp. Pure Math. 46 (1987), 199-222. 
[8] M. Cornalba: On the projectivity on the moduli spaces of curves, J. reine angew. Math. 443 (1993), 11-20.

[9] M. Culler and K. Vogtmann: Moduli of graphs and automorphisms of free groups, Invent. Math. 84 (1986), 91-119.

[10] M. Dehn: Die Gruppe der Abbildungsklassen, Acta Math. 69 (1938), 135-206.

[11] S. Diaz: A bound on the dimensions of complete subvarieties of $\mathcal{M}_{g}$, Duke Math. J. 51 (1984), 405-408.

[12] P. Deligne and D. Mumford: The irreducibility of the space of curves of given genus Inst. Hautes Études Sci. Publ. Math. 36 (1969), 75-109.

[13] C. Faber: Chow rings of moduli spaces of curves I,II, Ann. of Math. 132 (1990), 331-449.

[14] C. Faber: The tautological ring of $\mathcal{M}_{g}$ is one-dimensional in degree $g-2$, preprint Univ. van Amsterdam (1995).

[15] J. Foisy: The second homology group of the level 2 mapping class group of an orientable surface, Ph.D. Thesis, Duke University, Spring, 1996.

[16] S. Gervais: Presentation and central extensions of mapping class groups, preprint Univ. de Nantes (1995), to appear in the Trans. Am. Math. Soc.

[17] E. Getzler: Operads and moduli spaces of genus 0 Riemann surfaces, in The moduli space of curves, R. Dijkgraaf, C. Faber, G. van der Geer, editors, Birkhäuser, 1995, 199-230.

[18] V. Ginzburg and M. Kapranov: Koszul duality for operads Duke Math. J. 76 (1994), 203-272.

[19] A. Grothendieck: La longue marche à travers de la théorie de Galois, Manuscript written in 1981, to appear. (First few chapters obtainable from malgoire@math.univ.montp2.fr.)

[20] A. Grothendieck: Esquisse d'un programme, 1984 manuscript, will appear in a book edited by L. Schneps and P. Lochak and to be published by Cambridge UP.

[21] A. Grothendieck: The Grothendieck theory of dessins d'enfants, L. Schneps, editor, London Math. Soc. Lecture Note Series 200 (1994), Cambridge University Press.

[22] R. Hain: Algebraic cycles and extensions of variations of mixed Hodge structure, Proc. Symp. Pure Math. 53 (1991), 175-221.

[23] R. Hain: Completions of mapping class groups and the cycle $C-C^{-}$, in Mapping Class Groups and Moduli Spaces of Riemann Surfaces, C.-F. Bödigheimer and R. Hain, editors, Contemp. Math. 150 (1993), 75-105.

[24] R. Hain: Torelli groups and Geometry of Moduli Spaces of Curves, in Current Topics in Complex Algebraic Geometry (C. H. Clemens and J. Kollar, eds.) MSRI publications no. 28, Cambridge University Press, 1995, 97-143.

[25] R. Hain: The Hodge de Rham Theory of Relative Malcev Completion, preprint, 1995. (Available from http://www.math.duke.edu/faculty/hain/)

[26] R. Hain: Infinitesimal presentations of the torelli groups, preprint, 1995. (Available from http://www .math.duke.edu/faculty/hain/)

[27] J. Harer: The second homology group of the mapping class group of an orientable surface, Invent. Math. 72 (1983), 221-239.

[28] J. Harer: The virtual cohomology dimension of the mapping class group of an orientable surface, Invent. Math. 84 (1986), 157-176.

[29] J. Harer: Stability of the homology of the mapping class groups of orientable surfaces, Ann. of Math. 121 (1985), 215-129.

[30] J. Harer: The third homology group of the moduli space of curves, Duke J. Math. 63 (1992), $25-55$.

[31] J. Harer: Improved stability for the homology of the mapping class groups of of surfaces, Duke University preprint, 1993.

[32] J. Harer: The fourth homology group of the moduli space of curves, preprint, 1993.

[33] J. Harer: The rational Picard group of the moduli spaces of Riemann surfaces with spin structure, in Mapping Class Groups and Moduli Spaces of Riemann Surfaces, C.-F. Bödigheimer and R. Hain, editors, Contemp. Math. 150 (1993), 107-136.

[34] J. Harer and D. Zagier: The Euler characteristic of the moduli space of curves, Invent. Math. 85 (1986), 457-485.

[35] N. Ivanov: Stabilization of the homology of Teichmüller groups, Leningrad Math. J. 1 (1989) Engl. transl.: 675-691.

[36] N. Ivanov: On the homology stability for Teichmüller modular groups: closed surfaces and twisted coefficients, in Mapping Class Groups and Moduli Spaces of Riemann Surfaces, C.F. Bödigheimer and R. Hain, editors, Contemp. Math. 150 (1993), 149-194. 
[37] N. Ivanov: Mapping class groups, to appear in Handbook in Geometric Topology, (R. J. Daverman and R. B. Sher, eds.) North-Holland, 1997.

[38] N. Ivanov: Homomorphisms from arithmetic to modular groups, in preparation.

[39] N. Ivanov, R. McCarthy: On injective homomorphisms between Teichmüller modular groups, Preprint, 1994, 85 pp.

[40] E. Izadi: The Chow ring of the moduli space of curves of genus 5, in The moduli space of curves, R. Dijkgraaf, C. Faber, G. van der Geer, editors, Birkhauser, 1995, 267-304.

[41] D. Johnson: An abelian quotient of the mapping class group $\mathcal{I}_{g}$, Math. Ann. 249 (1980), 225-242.

[42] D. Johnson: The structure of the Torelli group I: A finite set of generators for $\mathcal{I}$, Ann. of Math. 118 (1983), 423-442.

[43] D. Johnson: The structure of the Torelli group - II: A characterization of the group generated by twists on bounding curves, Topology 24 (1985), 113-126.

[44] D. Johnson: The structure of the Torelli group-III: The abelianization of $\mathcal{I}$, Topology 24 (1985), 127-144.

[45] D. Johnson: A survey of the Torelli group, Contemp. Math. 20 (1983), 165-179.

[46] A. Kabanov: The second cohomology of moduli spaces of Riemann surfaces with twisted coefficients, Thesis, Duke University, 1995.

[47] V. Kaimanovich, H. Masur: The Poisson boundary of the mapping class group, Preprint, 1996, 43 pp.

[48] N. Kawazumi, S. Morita: In preparation, 1996.

[49] S. Keel: Intersection theory of moduli spaces of stable $n$-pointed curves of genus zero, Trans. Amer. math. Soc. 330 (1992), 545-574.

[50] M. Kontsevich: Intersection theory and the moduli space of curves and the matrix Airy function, Comm. Math. Phys. 147 (1992), 1-23.

[51] M. Kontsevich: Formal (non-)commutative symplectic geometry, in The Gelfand mathematical seminars 1990-1992, 173-188, Birkhäuser Verlag, Basel (1993).

[52] M. Kontsevich: Feynman diagrams and low-dimensional topology, in: Proc. First Eur. Math. Congr. at Paris (1992), Vol. II, 97-121, Birkhäuser Verlag, Basel (1994).

[53] M. Kontsevich and Yu. Manin (with an appendix by R. Kaufmann): Quantum cohomology of a product, Invent. Math. 124 (1996), 313-340.

[54] E. Looijenga: Cohomology of $\mathcal{M}_{3}$ and $\mathcal{M}_{3}^{1}$, in Mapping Class Groups and Moduli Spaces of Riemann Surfaces, C.-F. Bödigheimer and R. Hain, editors, Contemp. Math. 150 (1993), 205-228.

[55] E. Looijenga: Smooth Deligne-Mumford compactifications by means of Prym-level structures, J. Alg. Geom., 3 (1994), 283-293.

[56] E. Looijenga: Cellular decompositions of compactified moduli spaces of pointed curves, in The moduli space of curves, R. Dijkgraaf, C. Faber, G. van der Geer, editors, Birkhauser, 1995, 369-400.

[57] E. Looijenga: On the tautological ring of $\mathcal{M}_{g}$, Invent. Math. 121 (1995), 411-419.

[58] E. Looijenga: Stable cohomology of the mapping class group with symplectic coefficients and of the universal Abel-Jacobi map, J. Alg. Geom. 5 (1996), 135-150.

[59] Yu. Manin: Generating functions in algebraic geometry and sums over trees in The moduli space of curves, R. Dijkgraaf, C. Faber, G. van der Geer, editors, Birkhäuser, 1995, 401-417.

[60] G. Mess: The Torelli groups for genus 2 and 3 surfaces, Topology 31 (1992), 775-790.

[61] E. Miller: The homology of the mapping class group, J. Diff. Geom. 24 (1986), 1-14.

[62] J. Morgan: The algebraic topology of smooth algebraic varieties, Publ. Math. IHES 48 (1978), 137-204; correction, Publ. Math. IHES 64 (1986), 185.

[63] S. Morita: Characteristic classes of surface bundles, Invent. Math. 90 (1987), 551-577.

[64] S. Morita: On the structure and the homology of the Torelli group, Proc. Japan Acad. 65 (1989), 147-150.

[65] S. Morita: Casson's invariant for homology 3-spheres and characteristic classes of surface bundles I, Topology 28 (1989), 305-323.

[66] S. Morita: On the structure of the Torelli group and the Casson invariant, Topology 30 (1991), 603-621.

[67] S. Morita: The extension of Johnson's homomorphism from the Torelli group to the mapping class group, Invent. Math. 111 (1993), 197-224. 
[68] S. Morita: Abelian quotients of subgroups of the mapping class group of surfaces, Duke Math. J. 70 (1993), 699-726.

[69] D. Mumford Towards an enumerative geometry of the moduli space of curves, in Arithmetic and Geometry, M. Artin and J. Tate, editors (1983), Birkhaüser Verlag (Basel) 271-328

[70] H. Nakamura: Coupling of universal monodromy representations of Galois-Teichmüller modular groups, Math. Ann. 304 (1996), 99-119.

[71] H. Nakamura: Galois rigidity of profinite fundamental groups, Sugaku 47 (1995), 1-17 (in Japanese). English translation to appear in Sugaku Exposition (AMS).

[72] J. Nielsen: Untersuchungen zur Topologie der geschlossenen zweiseitigen Flächen I, Acta Math. 50 (1927), 189-358.

[73] M. Pikaart: An orbifold partition of $\overline{\mathcal{M}}_{g}^{n}$, in The moduli space of curves, R. Dijkgraaf, C. Faber, G. van der Geer, editors, Birkhäuser, 1995, 467-482.

[74] M. Pikaart and A. de Jong: Moduli of curves with non-abelian level structure, in The moduli space of curves, R. Dijkgraaf, C. Faber, G. van der Geer, editors, Birkhäuser, 1995, 483-509.

[75] M. Ragunathan: Cohomology of arithmetic subgroups of algebraic groups: I, Ann. of Math. 86 (1967), 409-424.

[76] K. Strebel: Quadratic differentials, Springer, 1984.

[77] E. Witten: Two dimensional gravity and intersection theory on moduli space, Surveys in Diff. Geom. 1 (1991), 243-310.

[78] B. Waynryb: A simple presentation for the mapping class group of an oriented surface, Isr. J. Math. 45 (1983), 157-174.

Department of Mathematics, Duke University, Durham, NC 27708-0320

E-mail address: hain@math.duke.edu

Faculteit Wiskunde en Informatica, University of Utrecht, Postal Box 80.010, NL3508 TA Utrecht, The Netherlands

E-mail address: looijeng@math.ruu.nl 